\title{
Nutritional status of a nereidid polychaete cultured in sand filters of mariculture wastewater
}

\author{
P.J. PALMER ${ }^{1}$, S. WANG ${ }^{1}$, A. HOULIHAN ${ }^{2}$ \& I. BROCK ${ }^{2}$ \\ ${ }^{1}$ Department of Agriculture, Fisheries and Forestry (DAFF), Bribie Island Research Centre, Woorim, Qld, Australia; \\ ${ }^{2}$ Department of Agriculture, Fisheries and Forestry, Health and Food Sciences Precinct, Coopers Plains, Qld, Australia
}

\begin{abstract}
This study examined the nutritional composition of the intertidal marine polychaete Perinereis helleri (Nereididae) when artificially cultured in sand filters treating mariculture wastewater. Moisture levels in harvested P. helleri ranged from 758 to $855 \mathrm{~g} \mathrm{~kg}^{-1}$, and ash, from 23 to $61 \mathrm{~g} \mathrm{~kg}^{-1}$ wet matter (WM). Stocking density and graded size after harvest significantly affected their composition. Higher total lipid contents were found in large $(>0.6 \mathrm{~g}) \quad P$. helleri (16-19 $\mathrm{g} \mathrm{kg}^{-1} \mathrm{WM}$ ) and those grown at the lowest density $\left(1000 \mathrm{~m}^{-2}: 18 \mathrm{~g} \mathrm{~kg}^{-1} \mathrm{WM}\right)$ than in small $(\leq 0.6 \mathrm{~g})$ ones (14 $\left.\mathrm{g} \mathrm{kg}^{-1} \mathrm{WM}\right)$ and those grown at the highest densities (4000-6000 m-2: 13-16 $\mathrm{g} \mathrm{kg}^{-1} \mathrm{WM}$ ). Several fatty acids within a very broad profile (some 30 identified) reflected this pattern, yet their ARA/EPA/DHA ratios were relatively unaffected. Feeding the polychaete-assisted sand filters (PASF) with fish meal to increase worm biomass productivity significantly increased their DHA content. Other components (e.g. protein, phospholipids, cholesterol, carbohydrate, amino acids, nitrogen, minerals and bromophenols) and nutritional factors (e.g. maturity, feeding seaweed and endemic shrimp viral content) were also investigated. Results suggest that PASF-produced $P$. helleri have a well-balanced nutritional profile for penaeid shrimp and fish broodstock.
\end{abstract}

KEY WORDS: amino acids, biosecurity, broodstock nutrition, lipids, minerals, Nereididae

Received 30 April 2013; accepted 14 August 2013

Correspondence: P.J. Palmer, Bribie Island Research Centre, PO Box 2066,Woorim,Qld 4507,Australia.E-mail: paul.palmer@daff.qld.gov.au

\section{Introduction}

Nutritious diets for broodstock underpin successful operations for cultured species throughout the world. Maturation diets need to be palatable and digestible and provide sufficient energy and a balance of essential nutrients to facilitate high reproductive success. In particular, marine fish and shrimp broodstock are known to have requirements for a range of essential fatty acids which they cannot synthesize themselves (Izquierdo et al. 2001; Wouters et al. 2001). These have traditionally been supplied by lipids in live and fresh/frozen diets mainly comprising a range of marine organisms including squid, bivalve molluscs, crustaceans and polychaetes (Marsden et al. 1992; Meunpol et al. 2005; Hoa et al. 2009). Although polychaetes are the most expensive of these diets, they are widely considered indispensible in commercial penaeid maturation diets (Harrison 1990; Kawahigashi 1998). However, their supplies are still largely dependent on wild populations, so can be affected by overfishing and habitat disturbances (Scaps 2003). Also, their nutritional contents are known to vary according to seasonal and environmental conditions (Garcia-Alonso et al. 2008), and their biosecurity status has recently been questioned (Vijayan et al. 2005). These factors suggest that alternative feeds or alternative production strategies for suitable polychaete species are needed.

To improve reliability of content and supply for shrimp maturation, several researchers have turned to replacement or supplementation of live and fresh/frozen feeds with formulated feeds (Wouters et al. 2001). These have been basically modelled on the measured contents of shrimp broodstock (mature ovaries: Hoa et al. 2009) and some of their more common natural feeds (e.g. squid and mussel: Marsden et al. 1997; polychaetes: Meunpol et al. 2005). A few studies have incorporated polychaetes into manufactured feeds for targeted nutrition and their chemical attractant properties (Palmer \& Slattery 2009; Chung et al. 2011). However, in maturation practices, compounded diets are still almost always used in combination with fresh feeds (see Coman et al. 2007; Turchini et al. 2009), where intact polychaetes have remained an integral part of commercial 
shrimp broodstock nutrition. Their neutral lipids and longchain polyunsaturated fatty acids (lcPUFA) are thought to contribute significantly to gonadal maturation (Middleditch et al. 1980; Lytle et al. 1990; Nguyen et al. 2012). In addition, they have been found to contain several substances that directly stimulate the endocrine system in penaeid shrimp (e.g. steroids: Laufer et al. 1997; prostaglandin: Croz et al. 1988; Poltana et al. 2005; Meunpol et al. 2010; progesterone: Meunpol et al. 2007). These combined factors demonstrate the importance of reliable, biosecure polychaete supplies for future developments in the marine shrimp (and fish) farming industry.

The development of polychaete-assisted sand filters (PASF) for treating mariculture wastewater (Palmer 2010) has created the opportunity to produce large quantities of polychaetes from waste nutrients in land-based marine farming systems. The PASF system can be applied to wastewater from broad-scale mariculture ponds for onfarm recirculation or environmentally benign discharge. Its main actions are to capture the phytoplankton (and other suspended debris) that proliferate in the water column of outdoor semi-intensive cultures and present it in a metered way as food to polychaetes that are cultured in sand filtration beds. It can provide a functional link between particulate organic and inorganic nutrients in integrated systems and at large scale has recently been shown to significantly reduce suspended solids, total nitrogen and total phosphorus levels in wastewater at commercial shrimp and fish farms (DAFF, unpublished data). Given this desirable broad-scale water treatment amenity, its application may expand in the future where environmentally sustainable, biosecure seafood production systems and increasing amounts of polychaete biomass are sought.

To date, relatively small quantities of Perinereis helleri have been cultured in Queensland in conjunction with the development and testing of the PASF system. However, this product has successfully replaced marine bloodworms (Marphysa sp.) in fish and shrimp hatcheries and in fishing bait markets (P.J. Palmer, pers. obs.). Because its nutritional content and biosecurity status were important aspects for these uses, studies were undertaken regarding its proximate composition, fatty and amino acids and other potentially active nutritional compounds, as well as routine screening for endemic shrimp viruses. This article documents the nutritional status of $P$. helleri grown in functional PASF systems and investigates the ways that environmental factors and commercial imperatives may affect their most important nutritional attributes.

\section{Materials and methods}

\section{Experimental animals and sampling}

Several hundred mature $P$. helleri collected from Moreton Bay in South East Queensland (Long. $153^{\circ} \mathrm{E}$, Lat. $27^{\circ} \mathrm{S}$ ) in 2005 formed the initial breeding nucleus. These were spawned and incubated using proprietary methods. Onemonth-old cultured juveniles were stocked and grown in PASF systems at increasing scales of application from 2005 to 2010. Broodstock collected from PASF beds gave rise to subsequent generations each year.

To obtain polychaete biomass for analyses, random samples of the sand substrate were removed from PASF beds and worms within were separated from the sand using 2-mm sieves. Further separation from mucus-laden silt was undertaken on bulk using the worms' active movement through larger $(8-\mathrm{mm})$ sieves, and all worms were then purged en masse in clean sea water. Samples for nutritional content analyses were purged for $4 \mathrm{~h}$, sorted, drip-dried on bulk (20 $\mathrm{s}$ in a fine net), placed into plastic bags and frozen in 50 - to 100 -g lots by placing directly into a $-80{ }^{\circ} \mathrm{C}$ ultrafreezer. Each sorted sample constituted pools of several hundred worms in the specified categories. These were thawed and homogenized immediately prior to analysis.

\section{Biomass compositional analyses}

Two Queensland State Government laboratories undertook the majority of biomass analyses. In 2005 and 2006, official AOAC (2000) methods were used at the Innovative Food Technologies Laboratory at Hamilton, Brisbane. Proximate analyses involved moisture, total lipid (including free fatty acids, triacylglycerols and membrane-bound phospholipids), fat (hexane-soluble material extracted under reflux), total nitrogen, total phosphorous, protein (by calculation), ash, carbohydrate (by difference), cholesterol (after Kovacs et al. 1979) and energy (FSANZ Food Standards Code Vol 2) assessments. Phospholipid analyses used high-performance liquid chromatography calibrated using phosphatidylcholine, phosphatidylethanolamine, lysophosphatidylethanolamine and sphingomyelin. Bromophenols were assessed using stable isotope dilution analysis in combination with solid-phase microextraction according to the methods described by Fuller et al. (2008). Minerals were assessed by ALS Environmental, Brisbane, Australia, using emission spectrophotometry (ICP-AES).

From 2007 to 2010, biomass analyses were undertaken at the Animal Research Institute, Yeerongpilly, Brisbane. 
Lipids were extracted from the sample with chloroform/ methanol by the method of Folch et al. (1957). Total lipid was determined gravimetrically on an aliquot of the extract by drying for $4 \mathrm{~h}$ at $80^{\circ} \mathrm{C}$ in a preweighed glass vial. A further aliquot of the extract was taken for fatty acid analysis. The lipid fatty acids in the extract were derivitized to their fatty acid methyl esters (FAME) using 14\% boron trifluoride-methanol (Van Wijngaarden 1967). FAME were analysed on an Agilent Technologies 6890 gas chromatograph using split injection with helium carrier gas and a flame ionization detector. The column used was a DB23 fused-silica capillary column, $30 \mathrm{~m} \times 0.25 \mathrm{~mm}$, with a $0.25-\mu \mathrm{m}$ coating (J\&W Agilent Technologies, Melbourne, Vic., Australia). Column oven temperature was held at $140{ }^{\circ} \mathrm{C}$ for $5 \mathrm{~min}$ and then elevated at $3{ }^{\circ} \mathrm{C} \mathrm{min}^{-1}$ to $210{ }^{\circ} \mathrm{C}$ where it was held until all FAME of interest had been eluted. FAME were identified by comparing their retention times with those of authentic standards (SigmaAldrich Co, Sydney, NSW, Australia) and were quantified by comparison with the response of an internal standard, heneicosanoic acid. Unknown fatty acids were tentatively identified by GC-MS analysis of their 3-pyridylcarbinol esters (Christie 2003) using an Agilent Technologies 5973 Mass Selective Detector with electron-impact ionization at $70 \mathrm{eV}$.

An aliquot of the lipid extract was prepared for cholesterol analysis by removing the lipids by saponification and extracting the cholesterol into hexane (Okshima 2001). Cholesterol was then determined by reversed-phase HPLC using a Shimadzu LC-10 Avp fitted with a Luna 5- $\mu \mathrm{m}$ C18(2), $250 \times 4.6 \mathrm{~mm}$ column (Phenomenex, Lane Cove, NSW, Australia) with methanol at $1 \mathrm{~mL} \mathrm{~min}^{-1}$ as mobile phase. A PDA detector at $218 \mathrm{~nm}$ was used to quantify the cholesterol against an external standard (SigmaAldrich Co, Sydney, NSW, Australia). The phospholipid profile of the lipid extract was determined by normalphase HPLC using a Shimadzu LC-10 Avp fitted with a $4-\mu \mathrm{m}$ NovaPak silica $150 \times 3.9 \mathrm{~mm}$ column (Waters, Rydalmere, NSW, Australia) and an ELSD detector using the chromatographic conditions described by Avalli \& Contarini (2005). Phospholipids were quantified against a mixture of authentic standards (Sigma-Aldrich Co, Sydney, NSW, Australia).

Results were analysed with GenStat (2011) using one- or two-way ANOVA (with the replicate sand beds being the experimental units and pooled worms within beds taken as subsamples) and LSD pairwise comparison of means.

To also test PASF-grown P. helleri for endemic marine shrimp viruses, live worms were submitted for PCR testing at the Tropical and Aquatic Animal Health Laboratory, Biosecurity Queensland, Townsville, Australia. Samples submitted included pools selected at random from (i) PASF beds (concrete-raceway-based cultures) at the Bribie Island Research Centre (BIRC) in 2008 and 2010, (ii) several separate larger-scale PASF beds at a commercial Penaeus monodon farm in 2009 and (iii) four PASF beds at Farm A and single PASF beds at Farms B and C. In 2008, the pool of worms was purged in clean sea water for $4 \mathrm{~h}$ and shipped overnight to the laboratory. From 2009, worms were purged for $>24 \mathrm{~h}$ prior to shipping. Upon arrival at the laboratory, several worms in each pool were diced into 2- to 3-mm-long pieces, placed in nucleic acid stabilizer and homogenized to produce a supernatant for total nucleic acid extraction. These were tested for yellowhead virus (YHV)/gill-associated virus (GAV) using the IQ2000@ nested RT-PCR detection kit (GeneReach Biotechnology Corp., Taichung, Taiwan), for infectious haematopoietic and hypodermal necrosis virus (IHHNV) using the PCR method described by Tang et al. (2007) and for Mourilyan virus (MoV) using a RT-nested PCR method described by Cowley et al. (2005).

\section{Experimental designs}

Worm size and maturity In 2005, P. helleri grown in a PASF system housed within a $8.6-\mathrm{m}^{2}$ concrete raceway at BIRC were randomly sampled on three separate occasions to provide material for proximate analyses. On each occasion, harvested worms were visually sorted into small $(\leq 0.6 \mathrm{~g})$ and large $(>0.6 \mathrm{~g})$ individuals. This divide was based on observations that lucrative bait markets in Australia accept $P$. helleri down to a size of $0.6 \mathrm{~g}$. Because recreational fishers prefer to use the larger worms that are more easily hooked and smaller worms tend to be more easily spread between broodstock without the need for chopping (which can also foul the water in broodstock tanks), producers will likely grade harvested worms into these two size classes.

On the last sampling occasion in 2005, because the worms were reaching maturity, they were initially also separated into non-maturing and maturing groups, with maturing worms separated into males (red) and females (green), and non-maturing worms sorted for size as above.

Supplemental feeding with seaweed In 2006, P. helleri grown in PASF systems housed in experimental plastic tubs $\left(0.4 \mathrm{~m}^{2} ; 200 \mathrm{~L}\right)$ were studied. For some beds, a layer of the seaweed (Enteromorpha prolifera) which the worms avidly consumed was physically maintained over the surface of 
beds for 1 month prior to harvest. In this case, worms harvested from tubs (several hundred worms pooled from three tubs in each category) were not size sorted, and because samples were taken before the onset of final maturation, they were also not separated by sex.

Pooled samples of $P$. helleri taken from the raceway and tubs in 2005 and 2006 were also assessed for their minerals and bromophenols contents, and the proximate and minerals composition of the seaweed fed to the worms was also assessed.

Worm size and density Perinereis helleri harvested from two worm density experiments in 2007 were investigated for their proximate, fatty acid and amino acid compositions. Three stocking densities (1000, 2000 and $4000 \mathrm{~m}^{-2}$; $n=2$ ) were used in a tub-based (as described previously) trial, and two stocking densities (2000 and $6000 \mathrm{~m}^{2} ; n=3$ ) were used in a tank-based $\left(1.84 \mathrm{~m}^{2} ; 2000 \mathrm{~L}\right)$ trial. The tubbased experiment was undertaken at BIRC, and the tankbased experiment was undertaken at a commercial shrimp (P. monodon) farm. These experiments were undertaken simultaneously at the two sites and were again based on the standardized operations of PASF systems stocked with 1-month-old juveniles. The entire contents of each tub and tank were harvested after 4-month operation and sorted into small and large individuals as previous.

Supplemental feeding with fish meal An experiment performed in 2010 with focus on commercial PASF designs also provided worm biomass from different treatments for analyses. This trial used polyethylene plastic pond liners to form larger-scale sand filtration beds (approximately $60 \mathrm{~m}^{2}$ ) of various designs at two shrimp (P. monodon) farms (A and B) and one fish (Sillago ciliata) farm (C). Physical differences between beds at different farms involved a completely outdoor, low-profile design at Farm A (four beds studied), a shade-house-covered in-ground design at Farm B (one bed) and an outdoor concrete-tanksupported design at Farm C (one bed). All beds were again stocked with 1 -month-old P. helleri juveniles at $2000 \mathrm{~m}^{-2}$ and supplied daily with the farms' wastewater.

Two of the four beds at Farm A were provided with supplemental feed based on recommendations for marine worm culture by Poltana et al. (2007). Accordingly, fish meal was spread evenly over these beds on a daily basis using the rates described in Fig. 1. Worm biomass was harvested from one square metre of each bed after 107, 115 and 125 days at Farms A, B and C, respectively. In each case, the worms sampled were purged for $24 \mathrm{~h}$ in clean sea

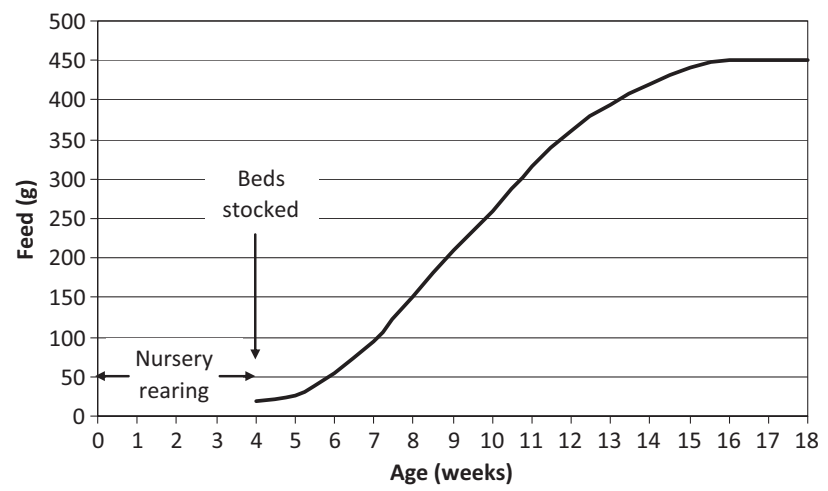

Figure 1 Daily fish meal feeding rates applied to two $60-\mathrm{m}^{2}$ beds at Farm A in 2010.

water at ambient temperature before freezing samples for nutritional analyses.

\section{Results}

\section{The effects of size grading, stocking density, maturity and feeding seaweed}

Small P. helleri grown in the concrete raceway in 2005 returned marginally higher $(P<0.05)$ moisture contents and lower $(P<0.02)$ fat and energy levels than large ones (Table 1). Mature females had comparatively low moisture levels and returned the highest fat contents for $P$. helleri samples analysed in 2005 and 2006. Females, and to a lesser extent males, also had relatively high levels of carbohydrate compared with small and large P. helleri. The proximate results for $P$. helleri grown in tubs without the seaweed layer did not differ greatly from those which had been feeding on E. prolifera, except in having somewhat elevated ash content.

There were few apparent differences between the mineral contents of $P$. helleri samples analysed in 2005 (Table 2). The lack of replication prevented statistical comparisons; however, the large pool of worms (several hundred) that constituted each sample meant that the results were robust. In general, it appeared that small worms had higher levels of sodium and zinc than large worms, whilst females returned higher levels of calcium, magnesium, potassium and sulphur than males and all other worm samples analysed. The worms were particularly high in sodium, potassium, sulphur, phosphorus (P), magnesium and calcium and, to a lesser extent, iron and zinc.

Large $P$. helleri tended to contain more bromophenols than small ones and had the highest total levels for the 
Table 1 Proximate composition ( $\mathrm{g} \mathrm{kg}^{-1}$ wet weight)* of Perinereis helleri grown in polychaete-assisted sand filters in 2005 and 2006

\begin{tabular}{|c|c|c|c|c|c|c|c|}
\hline Component & Small $^{1}$ & Large $^{1}$ & Male $^{2}$ & Female $^{2}$ & No seaweed ${ }^{3}$ & With seaweed ${ }^{3}$ & Seaweed $^{4}$ \\
\hline Moisture & $804.7 \pm 1.2^{\mathrm{a}}$ & $792 \pm 2.9^{b}$ & 807 & 758 & 790 & 814 & 890 \\
\hline $\mathrm{Fat}^{5}$ & $10.7 \pm 0.7^{\mathrm{b}}$ & $19.3 \pm 0.3^{a}$ & 12 & 36 & 15 & 17 & 5 \\
\hline Nitrogen & $21.5 \pm 0.1^{\mathrm{a}}$ & $21.3 \pm 0.3^{a}$ & 21.6 & 21.1 & 19.1 & 18.2 & 4.1 \\
\hline Protein $^{6}$ & $134.7 \pm 0.9^{\mathrm{a}}$ & $133 \pm 1.7^{\mathrm{a}}$ & 135 & 132 & 120 & 114 & 26 \\
\hline Ash & $38.7 \pm 0.7^{a}$ & $39.3 \pm 2.7^{a}$ & 23 & 32 & 61 & 37 & 43 \\
\hline Carbohydrate & $11.3 \pm 0.9^{\mathrm{a}}$ & $16 \pm 1.5^{a}$ & 23 & 42 & 14 & 18 & 36 \\
\hline Cholesterol & $1.85 \pm 0.22^{a}$ & $2.09 \pm 0.26^{a}$ & 1.98 & 2.41 & 1.30 & 1.18 & $<0.01$ \\
\hline Energy $^{7}$ & $2.88 \pm 0.01^{b}$ & $3.25 \pm 0.05^{a}$ & - & - & - & - & - \\
\hline
\end{tabular}

* Different superscripts within rows indicate significant $(P<0.05)$ differences.

${ }^{1}$ Means $( \pm$ SE) for non-maturing small $(\leq 0.6 \mathrm{~g})$ and large $(>0.6 \mathrm{~g})$ worms grown in raceway in $2005(n=3$ except for energy $n=2)$.

2 Male and female worms approaching maturity grown in raceway in $2005(n=1)$.

${ }^{3}$ Randomly selected worms grown in tubs with or without seaweed supplementation in $2006(n=1)$.

${ }^{4}$ Supplementary feed (Enteromorpha prolifera).

${ }^{5}$ Includes triglycerides, cholesterol and cholesterol esters.

${ }^{6}$ Total nitrogen $\times 6.25$.

${ }^{7} \mathrm{MJ} \mathrm{kg}{ }^{-1}$.

pooled samples studied (Table 2); 2,4-dibromophenol was the dominant form identified in all samples.

The moisture and ash contents of $P$. helleri biomass produced in 2007 in tub- and tank-based trials were not affected $(P>0.05)$ by density or graded size, with values similar to those found in 2005 and 2006. Mean dry matter contents were $203 \pm 3 \mathrm{~g} \mathrm{~kg}^{-1}$ for tubs and $182 \pm 6 \mathrm{~g} \mathrm{~kg}^{-1}$ for tanks (moisture in samples ranged from 777 to $\left.855 \mathrm{~g} \mathrm{~kg}^{-1}\right)$. The mean ash contents of harvested worms (WM) were $47 \pm 2 \mathrm{~g} \mathrm{~kg}^{-1}$ for tubs and $36 \pm 2 \mathrm{~g} \mathrm{~kg}^{-1}$ for tanks (samples ranged from 23 to $61 \mathrm{~g} \mathrm{~kg}^{-1}$ ).

Size had a pronounced $(P<0.005)$ effect on total lipid contents in both trials in 2007, where smaller worms consistently had lower lipid content (Table 3 ). Density also had a highly significant $(P<0.001)$ effect on total lipid content in the tub-based trial, but not in the tank-based trial $(P>0.05)$ (Table 3). This tendency for higher lipid content at lower densities was likely due to the worms growing to larger sizes at the lowest density.

Large worms had higher $(P<0.005)$ levels of energy than small worms in the tank-based trial, but in the tubbased trial, the two graded sizes were similar $(P>0.05)$ for this component (Table 3). Energy levels were also higher $(P<0.05)$ in worms harvested from the lowest stocking density used in the tub-based trial, and although a trend for decreasing energy with increasing densities was apparent, differences between the medium and high densities in both trials were not significant (Table 3).

Neither size nor density affected the levels of cholesterol $(P>0.05)$, where wet samples provided overall means $( \pm \mathrm{SE})$ of $1.37 \pm 0.03 \mathrm{~g} \mathrm{~kg}^{-1}$ in the tub-based trial and $0.95 \pm 0.02 \mathrm{~g} \mathrm{~kg}^{-1}$ in the tank-based trial. These values were somewhat lower than those found for $P$. helleri in 2005 and 2006. Marginally higher levels of nitrogen (N) and $\mathrm{P}$ were found in small compared with large worms in both trials, but these differences were only significant in the tub-based trial. In tubs, small worms (WM) had $21.8 \pm 0.2 \mathrm{~g} \mathrm{~kg}^{-1} \mathrm{~N}$ and $1.6 \pm 0 \mathrm{~g} \mathrm{~kg}^{-1} \mathrm{P}$, and large worms had $20.2 \pm 0.3 \mathrm{~g} \mathrm{~kg}^{-1} \mathrm{~N}$ and $1.5 \pm 0 \mathrm{~g} \mathrm{~kg}^{-1} \mathrm{P}$; in tanks, small worms had $19.5 \pm 0.8 \mathrm{~g} \mathrm{~kg}^{-1} \mathrm{~N}$ and $1.3 \pm 0.1 \mathrm{~g}$ $\mathrm{kg}^{-1} \mathrm{P}$, and large worms had $17.9 \pm 0.7 \mathrm{~g} \mathrm{~kg}^{-1} \mathrm{~N}$ and $1.2 \pm 0.1 \mathrm{~g} \mathrm{~kg}^{-1} \mathrm{P}$.

Neither size nor density affected $(P>0.05)$ total or specific phospholipid levels in $P$. helleri in 2007. Overall mean $( \pm \mathrm{SE}, n=12)$ levels (dry matter - DM) of total phospholipids were $19.6 \pm 1.2 \mathrm{mg} \mathrm{g}^{-1}$ in those from tubs and $13.7 \pm 2.2 \mathrm{mg} \mathrm{g}^{-1}$ in those from tanks. Mean levels in tubs and tanks for phosphatidylethanolamine were $8.3 \pm 0.5$ and $6.9 \pm 1.1 \mathrm{mg} \mathrm{g}^{-1}$, for phosphatidylinositol were $1.4 \pm 0.1$ and $0.9 \pm 0.3 \mathrm{mg} \mathrm{g}^{-1}$, for phosphatidylcholine were $8.8 \pm 0.7$ and $4 \pm 1.2 \mathrm{mg} \mathrm{g}^{-1}$ and for lysophosphatidylcholine were $1.2 \pm 0.2$ and $1.8 \pm 0.4 \mathrm{mg} \mathrm{g}^{-1}$, respectively. Phosphatidylserine and sphingomyelin were not detected in these analyses.

Palmitic acid (C16:0) was the most heavily represented fatty acid in $P$. helleri studies undertaken in 2007. It occurred at levels more than twice that of the next most heavily represented saturate, stearic acid (C18:0) (Table 4). Of those mono- and polyunsaturated fatty acids identified, 5,8,11,14,17-eicosapentaenoic (C20:5 $n$-3; EPA) was most heavily represented, followed closely by 7,10,13,16-docosatetraenoic acid (C22:4 $n$-6), and then cis-vaccenic (C18:1 $n$-7), arachidonic (C20:4 n-6; ARA), 5-octadecenoic (C18:1 $n$-13) and 7,10,13,16,19-docosapentaenoic (C22:5 n-3) acids. 
Table 2 Mineral $\left(\mathrm{mg} \mathrm{kg}^{-1}\right)$ and bromophenol $\left(\mathrm{ng} \mathrm{g}^{-1}\right)$ contents for pooled samples of Perinereis helleri grown in polychaete-assisted sand filters in 2005 and 2006. Data presented on a wet matter basis

\begin{tabular}{|c|c|c|c|c|c|c|c|}
\hline Mineral & Small $^{1}$ & Large $^{1}$ & Male $^{2}$ & Female $^{2}$ & Without seaweed ${ }^{3}$ & With seaweed ${ }^{3}$ & Seaweed $^{4}$ \\
\hline Aluminium & $<50$ & $<50$ & $<50$ & $<50$ & $<50$ & $<50$ & 160 \\
\hline Antimony & $<5$ & $<5$ & $<5$ & $<5$ & $<5$ & $<5$ & $<5$ \\
\hline Arsenic & 6 & 6 & 5 & 6 & $<5$ & $<5$ & $<5$ \\
\hline Barium & $<10$ & $<10$ & $<10$ & $<10$ & $<10$ & $<10$ & $<10$ \\
\hline Beryllium & $<1$ & $<1$ & $<1$ & $<1$ & $<1$ & $<1$ & $<1$ \\
\hline Boron & $<50$ & $<50$ & $<50$ & $<50$ & $<50$ & $<50$ & $<50$ \\
\hline Cadmium & $<1$ & $<1$ & $<1$ & $<1$ & $<1$ & $<1$ & $<1$ \\
\hline Calcium & 350 & 360 & 390 & 740 & 320 & 300 & 940 \\
\hline Chromium & $<2$ & $<2$ & $<2$ & $<2$ & 8 & 4 & $<2$ \\
\hline Cobalt & $<2$ & $<2$ & $<2$ & $<2$ & $<2$ & $<2$ & $<2$ \\
\hline Copper & $<5$ & $<5$ & 5 & $<5$ & $<5$ & 6 & $<5$ \\
\hline Iron & 100 & 90 & 60 & 60 & 200 & 130 & 1010 \\
\hline Lead & $<5$ & $<5$ & $<5$ & $<5$ & $<5$ & $<5$ & $<5$ \\
\hline Magnesium & 1020 & 950 & 810 & 1260 & 890 & 1040 & 2200 \\
\hline Manganese & $<5$ & $<5$ & 7 & 7 & $<5$ & $<5$ & 24 \\
\hline Molybdenum & $<2$ & $<2$ & $<2$ & $<2$ & $<2$ & $<2$ & $<2$ \\
\hline Nickel & $<2$ & $<2$ & $<2$ & $<2$ & 4 & $<2$ & $<2$ \\
\hline Phosphorus & 1700 & 1600 & 2020 & 1580 & 1370 & 1440 & 530 \\
\hline Potassium & 3200 & 3260 & 3250 & 4140 & 2860 & 2620 & 2910 \\
\hline Selenium & $<5$ & $<5$ & $<5$ & $<5$ & $<5$ & $<5$ & $<5$ \\
\hline Silver & $<2$ & $<2$ & $<2$ & $<2$ & $<2$ & $<2$ & $<2$ \\
\hline Sodium & 6190 & 5350 & 5130 & 4600 & 5210 & 5610 & 9780 \\
\hline Strontium & 6 & 5 & 5 & 6 & 5 & 5 & 13 \\
\hline Sulphur as $\mathrm{S}$ & 2690 & 2700 & 2510 & 4660 & 2150 & 2530 & 3360 \\
\hline Tin & $<5$ & $<5$ & $<5$ & $<5$ & $<5$ & $<5$ & $<5$ \\
\hline Titanium & $<10$ & $<10$ & $<10$ & $<10$ & $<10$ & $<10$ & $<10$ \\
\hline Vanadium & $<5$ & $<5$ & $<5$ & $<5$ & $<5$ & $<5$ & $<5$ \\
\hline Zinc & 48 & 29 & 12 & 20 & 29 & 20 & 9 \\
\hline \multicolumn{8}{|l|}{ Bromophenol } \\
\hline Total & 87.4 & 124.6 & - & - & 65.9 & - & - \\
\hline 2-bromophenol & 2.3 & 2.7 & - & - & 1.3 & - & - \\
\hline 4-bromophenol & 1 & 2.1 & - & - & 1.2 & - & - \\
\hline 2,4-dibromophenol & 69.5 & 96.4 & - & - & 46.3 & - & - \\
\hline 2,6-dibromophenol & 8.2 & 14.9 & - & - & 3.7 & - & - \\
\hline 2,4,6-tribromophenol & 6.4 & 8.5 & - & - & 13.4 & - & - \\
\hline
\end{tabular}

$<$ reading was below the limit of test as stated.

1 Small $(\leq 0.6 \mathrm{~g})$ or large $(>0.6 \mathrm{~g})$ worms grown in raceway in 2005 .

2 Male or female worms approaching maturity grown in raceway in 2005.

3 Randomly selected worms grown in tubs without or with seaweed supplementation in 2006.

${ }^{4}$ Supplementary feed (Enteromorpha prolifera).

Table 3 Mean $( \pm$ SE) total lipid and energy contents* of Perinereis helleri following size grading or grown at different stocking densities in polychaete-assisted sand filters in 2007. Data presented on a wet matter basis

\begin{tabular}{|c|c|c|c|c|c|c|}
\hline \multirow[b]{2}{*}{ Component } & \multirow[b]{2}{*}{ Culture environment } & \multicolumn{2}{|l|}{ Graded size $^{1}$} & \multicolumn{3}{|c|}{ Stocking density ${ }^{2}$} \\
\hline & & Small & Large & Low & Medium & High \\
\hline \multirow[t]{2}{*}{ Total lipid $\left(\mathrm{g} \mathrm{kg}^{-1}\right)$} & Tubs & $14.1 \pm 0.8^{\mathrm{b}}$ & $16.2 \pm 1.1^{\mathrm{a}}$ & $17.9 \pm 0.9^{\mathrm{a}}$ & $14.4 \pm 0.7^{b}$ & $13.1 \pm 0.5^{c}$ \\
\hline & Tanks & $13.5 \pm 0.5^{\mathrm{b}}$ & $18.6 \pm 1.1^{a}$ & - & $16.7 \pm 1.7^{\mathrm{a}}$ & $15.5 \pm 1.1^{\mathrm{a}}$ \\
\hline \multirow[t]{2}{*}{ Energy ( $\mathrm{MJ} \mathrm{kg}^{-1}$ ) } & Tubs & $3.73 \pm 0.06^{a}$ & $3.65 \pm 0.1^{\mathrm{a}}$ & $3.9 \pm 0.06^{\mathrm{a}}$ & $3.63 \pm 0.08^{\mathrm{b}}$ & $3.53 \pm 0.06^{b}$ \\
\hline & Tanks & $1.47 \pm 0.08^{b}$ & $1.99 \pm 0.09^{a}$ & - & $1.75 \pm 0.16^{\mathrm{a}}$ & $1.72 \pm 0.12^{\mathrm{a}}$ \\
\hline
\end{tabular}

* Within graded size or stocking density, different superscripts within rows indicate significant $(P<0.05)$ differences.

${ }^{1} n=6$ for small $(\leq 0.6 \mathrm{~g})$ or large $(>0.6 \mathrm{~g})$ worms.

${ }^{2} n=4$ for tubs at low $\left(1000 \mathrm{~m}^{-2}\right)$, medium $\left(2000 \mathrm{~m}^{-2}\right)$ and high $\left(4000 \mathrm{~m}^{-2}\right)$ densities; $n=6$ for tanks at medium $\left(2000 \mathrm{~m}^{-2}\right)$ and high $\left(6000 \mathrm{~m}^{-2}\right)$ densities. 
Table 4 Mean $( \pm$ SE, $n=6)$ fatty acid contents* $\left(\mathrm{mg} \mathrm{g}^{-1}\right)$ of small $(\leq 0.6 \mathrm{~g})$ and large $(>0.6 \mathrm{~g})$ Perinereis helleri grown in polychaete-assisted sand filters in 2007. Data presented on a dry matter basis

\begin{tabular}{|c|c|c|c|}
\hline Fatty acid & Culture environment & Small & Large \\
\hline \multicolumn{4}{|l|}{ Saturates } \\
\hline \multirow[t]{2}{*}{ C14:0 } & Tubs & $0.14 \pm 0.03^{b}$ & $0.21 \pm 0.01^{\mathrm{a}}$ \\
\hline & Tanks & $0.16 \pm 0.01^{\mathrm{b}}$ & $0.28 \pm 0.02^{\mathrm{a}}$ \\
\hline \multirow[t]{2}{*}{ C15:0 } & Tubs & $0.2 \pm 0.02^{b}$ & $0.29 \pm 0.01^{a}$ \\
\hline & Tanks & $0.16 \pm 0.01^{b}$ & $0.25 \pm 0.02^{\mathrm{a}}$ \\
\hline \multirow[t]{2}{*}{ C16:0 $0^{\text {palmitic }}$} & Tubs & $4.01 \pm 0.64^{b}$ & $7.87 \pm 0.72^{\mathrm{a}}$ \\
\hline & Tanks & $5.36 \pm 0.38^{* *}$ & $12.38 \pm 0.87$ ** \\
\hline \multirow[t]{2}{*}{$\mathrm{C} 17: 0$} & Tubs & $0.78 \pm 0.05^{\mathrm{a}}$ & $1.16 \pm 0.04^{b}$ \\
\hline & Tanks & $0.67 \pm 0.03^{b}$ & $0.98 \pm 0.05^{a}$ \\
\hline \multirow[t]{2}{*}{ C18:0 $0^{\text {stearic }}$} & Tubs & $2.27 \pm 0.17^{b}$ & $2.88 \pm 0.12^{\mathrm{a}}$ \\
\hline & Tanks & $2.45 \pm 0.12^{\mathrm{b}}$ & $3.48 \pm 0.16^{\mathrm{a}}$ \\
\hline \multirow[t]{2}{*}{$\mathrm{C} 20: 0$} & Tubs & $0.05 \pm 0.02 * *$ & $0.06 \pm 0 * *$ \\
\hline & Tanks & $0.07 \pm 0^{a}$ & $0.08 \pm 0^{a}$ \\
\hline \multirow[t]{2}{*}{$\mathrm{C} 24: 0$} & Tubs & $0.03 \pm 0.02^{a}$ & $0.03 \pm 0.02^{\mathrm{a}}$ \\
\hline & Tanks & $0.06 \pm 0.01^{\mathrm{a}}$ & $0.07 \pm 0.01^{\mathrm{a}}$ \\
\hline \multicolumn{4}{|l|}{ Mono- and polyunsaturates } \\
\hline \multirow[t]{2}{*}{ C16:1 n-7 } & Tubs & $0.54 \pm 0.11^{\mathrm{b}}$ & $0.85 \pm 0.12^{\mathrm{a}}$ \\
\hline & Tanks & $0.8 \pm 0.07^{\mathrm{b}}$ & $1.57 \pm 0.11^{\mathrm{a}}$ \\
\hline \multirow[t]{2}{*}{$\mathrm{C} 18: 1 n-13^{1}$} & Tubs & $0.94 \pm 0.08^{b}$ & $1.62 \pm 0.12^{\mathrm{a}}$ \\
\hline & Tanks & $1.25 \pm 0.07 * *$ & $2.52 \pm 0.17 * *$ \\
\hline \multirow{2}{*}{ C18:1 $n-7^{\text {cis-vaccenic }}$} & Tubs & $1.34 \pm 0.16^{\mathrm{b}}$ & $2.41 \pm 0.15^{\mathrm{a}}$ \\
\hline & Tanks & $1.91 \pm 0.14^{\mathrm{b}}$ & $3.64 \pm 0.24^{\mathrm{a}}$ \\
\hline \multirow[t]{2}{*}{ C18:1 n-9 } & Tubs & $0.47 \pm 0.08^{b}$ & $0.7 \pm 0.08^{a}$ \\
\hline & Tanks & $0.61 \pm 0.05^{\mathrm{b}}$ & $1.15 \pm 0.07^{\mathrm{a}}$ \\
\hline \multirow[t]{2}{*}{ C18:2 n-6 } & Tubs & $0.5 \pm 0.05^{\mathrm{a}}$ & $0.54 \pm 0.05^{\mathrm{a}}$ \\
\hline & Tanks & $0.61 \pm 0.03^{b}$ & $0.85 \pm 0.04^{\mathrm{a}}$ \\
\hline \multirow[t]{2}{*}{ C18:3 n-3 } & Tubs & $0.19 \pm 0.05^{b}$ & $0.24 \pm 0.07^{a}$ \\
\hline & Tanks & $0.46 \pm 0.06^{\mathrm{b}}$ & $0.79 \pm 0.1^{a}$ \\
\hline \multirow[t]{2}{*}{ C20:1 n-7 } & Tubs & $0.09 \pm 0.02^{b}$ & $0.12 \pm 0.01^{\mathrm{a}}$ \\
\hline & Tanks & $0.14 \pm 0.01^{b}$ & $0.19 \pm 0.01^{a}$ \\
\hline \multirow[t]{2}{*}{$C 20: 1 n-9$} & Tubs & $0.36 \pm 0.09^{b}$ & $0.65 \pm 0.08^{a}$ \\
\hline & Tanks & $0.48 \pm 0.04^{b}$ & $0.97 \pm 0.06^{\mathrm{a}}$ \\
\hline \multirow[t]{2}{*}{$\mathrm{C} 20: 1 n-11$} & Tubs & $0.5 \pm 0.04^{b}$ & $0.8 \pm 0.04^{a}$ \\
\hline & Tanks & $0.67 \pm 0.04^{b}$ & $1.28 \pm 0.08^{a}$ \\
\hline \multirow[t]{2}{*}{$5,11-C 20: 2^{2}$} & Tubs & $0.19 \pm 0.05^{b}$ & $0.44 \pm 0.08^{\mathrm{a}}$ \\
\hline & Tanks & $0.36 \pm 0.04^{b}$ & $0.94 \pm 0.06^{\mathrm{a}}$ \\
\hline$C 20: 2 n-6$ & Tubs & $0.32 \pm 0.06^{\mathrm{b}}$ & $0.56 \pm 0.08^{a}$ \\
\hline & Tanks & $0.45 \pm 0.03^{b}$ & $0.98 \pm 0.07^{\mathrm{a}}$ \\
\hline$C 20: 3 n-3$ & Tubs & $0 \pm 0 * *$ & $0.06 \pm 0.02 * *$ \\
\hline & Tanks & $0.04 \pm 0.01^{b}$ & $0.2 \pm 0.03^{a}$ \\
\hline C20:4 $n-6^{\text {ARA }}$ & Tubs & $1.57 \pm 0.07^{\mathrm{a}}$ & $1.55 \pm 0.06^{\mathrm{a}}$ \\
\hline & Tanks & $1.21 \pm 0.05^{b}$ & $1.63 \pm 0.07^{\mathrm{a}}$ \\
\hline $\mathrm{C} 20: 5 n-3^{\mathrm{EPA}}$ & Tubs & $3.08 \pm 0.13^{* *}$ & $3.86 \pm 0.07 * *$ \\
\hline & Tanks & $3.81 \pm 0.17^{\mathrm{a}}$ & $4.62 \pm 0.18^{a}$ \\
\hline$C 22: 1 n-9$ & Tubs & $0.02 \pm 0.01^{b}$ & $0.06 \pm 0.01^{\mathrm{a}}$ \\
\hline & Tanks & $0.01 \pm 0.01^{\mathrm{b}}$ & $0.08 \pm 0.01^{a}$ \\
\hline $7,13-C 22: 2^{3}$ & Tubs & $1.52 \pm 0.06^{\mathrm{b}}$ & $2.02 \pm 0.04^{a}$ \\
\hline & Tanks & $1.93 \pm 0.06^{b}$ & $2.65 \pm 0.06^{\mathrm{a}}$ \\
\hline C22:4 n-6 $7,10,13,16$-docosatetraenoic & Tubs & $3 \pm 0.05^{b}$ & $3.66 \pm 0.11^{a}$ \\
\hline & Tanks & $2.4 \pm 0.11^{b}$ & $3.17 \pm 0.13^{\mathrm{a}}$ \\
\hline C22:5 n-3 $3^{7,10,13,16,19-d o c o s a p e n t a e n o i c ~}$ & Tubs & $0.76 \pm 0.02^{\mathrm{b}}$ & $1.14 \pm 0.05^{\mathrm{a}}$ \\
\hline & Tanks & $0.94 \pm 0.05^{b}$ & $1.17 \pm 0.05^{\mathrm{a}}$ \\
\hline$C 22: 5 n-6$ & Tubs & $0.24 \pm 0.01^{\mathrm{b}}$ & $0.31 \pm 0.01^{\mathrm{a}}$ \\
\hline & Tanks & $0.12 \pm 0.01^{\mathrm{a}}$ & $0.15 \pm 0.01^{a}$ \\
\hline $\mathrm{C} 22: 6 n-3^{\mathrm{DHA}}$ & Tubs & $0.23 \pm 0.01^{b}$ & $0.31 \pm 0.01^{\mathrm{a}}$ \\
\hline & Tanks & $0.4 \pm 0.02^{b}$ & $0.55 \pm 0.04^{\mathrm{a}}$ \\
\hline
\end{tabular}


Table 4 (Continued)

\begin{tabular}{llll}
\hline Fatty acid & Culture environment & Small & Large \\
\hline C24:1 $n-9$ & Tubs & $0.02 \pm 0.01^{* *}$ & $0.07 \pm 0 * *$ \\
& Tanks & $0.01 \pm 0.01^{\mathrm{b}}$ & $0.05 \pm 0.01^{\mathrm{a}}$ \\
ARA : EPA : DHA ratio & Tubs & $1: 2: 0.1$ & $1: 2.5: 0.2$ \\
& Tanks & $1: 3.1: 0.3$ & $1: 2.8: 0.3$ \\
\hline
\end{tabular}

* Different superscripts within rows indicate significant $(P<0.05)$ differences.

** Significant $(P<0.05)$ interaction between density and size; see Table 6.

${ }^{1}$ Tentatively identified as cis-5-octadecenoic acid.

2 Tentatively identified cis-5, cis-11-eicosadienoic acid.

${ }^{3}$ Tentatively identified as cis-7,cis-13-docosadienoic acid.

In almost all cases, large worms had higher levels of specific fatty acids than small worms. Similarly, in most cases, worms grown at the lowest densities had higher levels of specific saturated and mono-/polyunsaturated fatty acids (Table 5). Exceptions were 7,10,13,16-docosatetraenoic, 7,10,13,16,19-docosapentaenoic and 4,7,10,13,16-docosapentaenoic (C22:5 n-6) acids, which were each detected at similar $(P>0.05)$ levels in all densities compared in 2007.

There were significant interactions between density and size for EPA, 11,14,17-eicosatrienoic (C20:3n-3), nervonic (C24:1 n-9) and arachidic (C20:0) acids in the tub-based trial and for 5-octadecenoic and palmitic acids in the tank-based trial in 2007 (see Table 6). Differences between small and large worms were generally more pronounced in the higher densities where greater competition slowed the growth. The ARA/EPA/DHA ratios showed little change across the 2007 trials (see Tables 4 and 5) due to the similar proportional effects of factors on each of these fatty acids. However, compared with those of tubs, tank-based conditions did generally appear to generate higher EPA levels.

Other fatty acids that were detected at low levels, but not affected $(P>0.05)$ by either size or density in tub- and tank-based experiments in 2007 (not shown in Tables 4-6) included octadecatetraenoic acid (C18:4 n-3) (0.03 \pm 0.01 and $\left.0.15 \pm 0.02 \mathrm{mg} \mathrm{g}^{-1}\right)$ and behenic acid (C22:0) $\left(0.01 \pm 0.01\right.$ and $\left.0.03 \pm 0.01 \mathrm{mg} \mathrm{g}^{-1}\right)$ (means $\pm \mathrm{SE}$ for tubs and tanks provided, respectively).

\section{The effect of supplemental feeding with fish meal}

Supplemental feeding with fish meal had few significant effects on the proximate composition of $P$. helleri. It did not affect $(P>0.05)$ their levels of moisture or ash at Farm A (779-792 $\mathrm{g} \mathrm{kg}^{-1} \mathrm{WM}$ and $105-118 \mathrm{~g} \mathrm{~kg}^{-1} \mathrm{DM}$, respectively), which were slightly lower than at the other farms (Table 7). Differences in energy contents of $P$. helleri between fed and unfed beds at Farm A were close to signif- icant $(P=0.053)$, with fed beds demonstrating the highest levels amongst all farms. Fat and total lipid levels at Farm A (73-87 and $127-130 \mathrm{~g} \mathrm{~kg}^{-1}$, respectively) were also not significantly affected by feeding the worms fish meal, but were somewhat higher than levels returned for worms from Farms B and C. Similar levels of cholesterol prevailed in $P$. helleri across all three farms, although those from unfed beds at Farm A did have significantly higher levels than from fed beds. The $\mathrm{N}$ and $\mathrm{P}$ levels of worms at Farm $\mathrm{A}$ (104 and 7.7-7.8 $\mathrm{g} \mathrm{kg}^{-1}$, respectively) were not significantly affected by this supplemental feeding and were quite similar to levels detected at Farms B and C (Table 7).

Levels of most saturated fatty acids were similar in fed and unfed beds. Exceptions included the most prevalent palmitic acid, which was higher $(P<0.05)$ in fed beds, and behenic acid, which was identified at low levels in fed beds, but was below the detection limits in all unfed beds (including beds at Farms $\mathrm{B}$ and $\mathrm{C}-$ see Table 8). For the 22 mono- and polyunsaturates detected, ten were found at similar $(P>0.05)$ levels in fed and unfed beds, and the remainder were significantly higher in fed beds except for 9-eicosenoic (C20:1 n-11), 7,10,13,16-docosatetraenoic and 7,10,13,16,19-docosapentaenoic acids which were significantly higher in unfed beds. Levels of DHA were particularly enhanced by feeding fish meal, where mean levels in $P$. helleri from fed beds were more than four times greater than those from unfed beds. The ARA/EPA/DHA ratios were similar for $P$. helleri sampled from Farms B and C, but for similarly unfed beds at Farm A, the EPA ratio was comparatively elevated. Levels of most other fatty acids in $P$. helleri from unfed beds at Farm A were also well above those found at the other two (unfed) farms; exceptions were arachidic, behenic, linoleic (C18-2 n-6), ARA, docosadienoic (7,13-22:2), 7,10,13,16-docosatetraenoic and nervonic acids.

Of the 18 amino acids studied in 2010 (Table 9), only four were significantly affected by supplemental feeding with fish 
Table 5 Mean $( \pm \mathrm{SE})$ fatty acid contents* $\left(\mathrm{mg} \mathrm{g}^{-1}\right)$ of Perinereis helleri grown at different stocking densities in polychaete-assisted sand filters in 2007. Data presented on a dry matter basis

\begin{tabular}{|c|c|c|c|c|}
\hline Fatty acid & Culture environment & Low density & Medium density & High density \\
\hline \multicolumn{5}{|l|}{ Saturates } \\
\hline \multirow[t]{2}{*}{ C14:0 } & Tubs $^{1}$ & $0.23 \pm 0.02^{a}$ & $0.16 \pm 0.02^{\mathrm{b}}$ & $0.13 \pm 0.03^{c}$ \\
\hline & Tanks $^{2}$ & - & $0.25 \pm 0.03^{a}$ & $0.19 \pm 0.02^{\mathrm{b}}$ \\
\hline \multirow[t]{2}{*}{$\mathrm{C} 15: 0$} & Tubs $^{1}$ & $0.28 \pm 0.02^{a}$ & $0.23 \pm 0.03^{b}$ & $0.22 \pm 0.03^{b}$ \\
\hline & Tanks $^{2}$ & - & $0.22 \pm 0.03^{a}$ & $0.19 \pm 0.02^{a}$ \\
\hline \multirow[t]{2}{*}{$\mathrm{C} 16: 0$} & Tubs $^{1}$ & $7.98 \pm 1.16^{\mathrm{a}}$ & $5.45 \pm 1.17^{b}$ & $4.4 \pm 1.05^{c}$ \\
\hline & Tanks $^{2}$ & - & $10.15 \pm 1.64^{*}$ & $7.59 \pm 1.34^{*}$ \\
\hline \multirow[t]{2}{*}{$\mathrm{C} 17: 0$} & Tubs $^{1}$ & $1.08 \pm 0.09^{a}$ & $0.96 \pm 0.11^{b}$ & $0.87 \pm 0.13^{b}$ \\
\hline & Tanks $^{2}$ & - & $0.89 \pm 0.1^{a}$ & $0.76 \pm 0.07^{\mathrm{b}}$ \\
\hline \multirow[t]{2}{*}{ C18:0 } & Tubs $^{1}$ & $3.01 \pm 0.14^{\mathrm{a}}$ & $2.47 \pm 0.18^{\mathrm{b}}$ & $2.25 \pm 0.23^{c}$ \\
\hline & Tanks $^{2}$ & - & $3.25 \pm 0.24^{\mathrm{a}}$ & $2.69 \pm 0.21^{b}$ \\
\hline \multirow[t]{2}{*}{$\mathrm{C} 20: 0$} & Tubs $^{1}$ & $0.08 \pm 0 * *$ & $0.06 \pm 0 * *$ & $0.03 \pm 0.02 * *$ \\
\hline & Tanks $^{2}$ & - & $0.08 \pm 0^{\mathrm{a}}$ & $0.06 \pm 0^{\mathrm{b}}$ \\
\hline \multirow[t]{2}{*}{$\mathrm{C} 24: 0$} & Tubs $^{1}$ & $0.08 \pm 0.01^{\mathrm{a}}$ & $0 \pm 0^{\mathrm{b}}$ & $0 \pm 0^{\mathrm{b}}$ \\
\hline & Tanks $^{2}$ & - & $0.08 \pm 0^{\mathrm{a}}$ & $0.04 \pm 0.01^{b}$ \\
\hline \multicolumn{5}{|l|}{ Mono- and polyunsaturates } \\
\hline \multirow[t]{2}{*}{ C16:1 n-7 } & Tubs $^{1}$ & $1.03 \pm 0.11^{\mathrm{a}}$ & $0.59 \pm 0.1^{\mathrm{b}}$ & $0.46 \pm 0.08^{\mathrm{b}}$ \\
\hline & Tanks $^{2}$ & - & $1.36 \pm 0.17^{\mathrm{a}}$ & $1.01 \pm 0.16^{\mathrm{b}}$ \\
\hline \multirow[t]{2}{*}{ C18:1 n-7 } & Tubs $^{1}$ & $2.3 \pm 0.29^{a}$ & $1.82 \pm 0.32^{\mathrm{b}}$ & $1.51 \pm 0.34^{c}$ \\
\hline & Tanks $^{2}$ & - & $3.17 \pm 0.46^{a}$ & $2.39 \pm 0.35^{\mathrm{b}}$ \\
\hline \multirow[t]{2}{*}{ C18:1 n-9 } & Tubs $^{1}$ & $0.82 \pm 0.07^{\mathrm{a}}$ & $0.52 \pm 0.06^{\mathrm{b}}$ & $0.41 \pm 0.08^{c}$ \\
\hline & Tanks $^{2}$ & - & $0.99 \pm 0.11^{\mathrm{a}}$ & $0.77 \pm 0.12^{\mathrm{b}}$ \\
\hline \multirow[t]{2}{*}{ C18:1 n-13 } & Tubs $^{1}$ & $1.58 \pm 0.23^{\mathrm{a}}$ & $1.2 \pm 0.2^{\mathrm{b}}$ & $1.1 \pm 0.17^{\mathrm{b}}$ \\
\hline & Tanks $^{2}$ & - & $2.14 \pm 0.31 * *$ & $1.63 \pm 0.24$ ** \\
\hline \multirow[t]{2}{*}{ C18:2 n-6 } & Tubs $^{1}$ & $0.66 \pm 0.01^{\mathrm{a}}$ & $0.46 \pm 0.03^{b}$ & $0.43 \pm 0.02^{b}$ \\
\hline & Tanks $^{2}$ & - & $0.77 \pm 0.03^{\mathrm{a}}$ & $0.69 \pm 0.06^{\mathrm{a}}$ \\
\hline \multirow[t]{2}{*}{ C18:3 n-3 } & Tubs $^{1}$ & $0.4 \pm 0.04^{a}$ & $0.15 \pm 0.02^{b}$ & $0.1 \pm 0.01^{b}$ \\
\hline & Tanks $^{2}$ & - & $0.73 \pm 0.03^{a}$ & $0.52 \pm 0.08^{a}$ \\
\hline \multirow{2}{*}{ C20:1 n-7 } & Tubs $^{1}$ & $0.14 \pm 0.01^{a}$ & $0.11 \pm 0.01^{\mathrm{b}}$ & $0.07 \pm 0.01^{c}$ \\
\hline & Tanks $^{2}$ & - & $0.18 \pm 0.02^{a}$ & $0.14 \pm 0.01^{b}$ \\
\hline \multirow[t]{2}{*}{$C 20: 1 n-9$} & Tubs $^{1}$ & $0.77 \pm 0.08^{\mathrm{a}}$ & $0.43 \pm 0.09^{b}$ & $0.32 \pm 0.1^{c}$ \\
\hline & Tanks $^{2}$ & - & $0.83 \pm 0.11^{\mathrm{a}}$ & $0.62 \pm 0.1^{\mathrm{b}}$ \\
\hline \multirow[t]{2}{*}{$\mathrm{C} 20: 1 n-11$} & Tubs $^{1}$ & $0.72 \pm 0.09^{a}$ & $0.67 \pm 0.09^{\mathrm{ab}}$ & $0.56 \pm 0.1^{\mathrm{b}}$ \\
\hline & Tanks $^{2}$ & - & $1.09 \pm 0.16^{a}$ & $0.85 \pm 0.12^{\mathrm{b}}$ \\
\hline $5,11-C 20: 2$ & Tubs $^{1}$ & $0.51 \pm 0.09^{a}$ & $0.25 \pm 0.06^{\mathrm{b}}$ & $0.18 \pm 0.06^{c}$ \\
\hline & Tanks $^{2}$ & - & $0.72 \pm 0.12^{a}$ & $0.57 \pm 0.12^{b}$ \\
\hline$C 20: 2 n-6$ & Tubs $^{1}$ & $0.65 \pm 0.09^{a}$ & $0.38 \pm 0.07^{b}$ & $0.29 \pm 0.06^{c}$ \\
\hline & Tanks $^{2}$ & - & $0.81 \pm 0.11^{a}$ & $0.62 \pm 0.11^{\mathrm{b}}$ \\
\hline$C 20: 3 n-3$ & Tubs $^{1}$ & $0.07 \pm 0.04^{* *}$ & $0.03 \pm 0.02 * *$ & $0 \pm 0 * *$ \\
\hline & Tanks $^{2}$ & - & $0.16 \pm 0.02^{\mathrm{a}}$ & $0.08 \pm 0.03^{b}$ \\
\hline C20:4 $n-6^{\text {ARA }}$ & Tubs $^{1}$ & $1.7 \pm 0.02^{\mathrm{a}}$ & $1.53 \pm 0.09^{\mathrm{ab}}$ & $1.44 \pm 0.03^{b}$ \\
\hline & Tanks $^{2}$ & - & $1.52 \pm 0.08^{a}$ & $1.31 \pm 0.09^{b}$ \\
\hline $\mathrm{C} 20: 5 n-3^{\mathrm{EPA}}$ & Tubs $^{1}$ & $3.61 \pm 0.11^{* *}$ & $3.54 \pm 0.23^{* *}$ & $3.27 \pm 0.35^{* *}$ \\
\hline & Tanks $^{2}$ & - & $4.46 \pm 0.32^{a}$ & $3.98 \pm 0.17^{a}$ \\
\hline$C 22: 1 n-9$ & Tubs $^{1}$ & $0.08 \pm 0.01^{a}$ & $0.03 \pm 0.02^{\mathrm{b}}$ & $0.02 \pm 0.02^{\mathrm{b}}$ \\
\hline & Tanks $^{2}$ & - & $0.05 \pm 0.02^{\mathrm{a}}$ & $0.03 \pm 0.02^{a}$ \\
\hline $7,13-C 22: 2$ & Tubs $^{1}$ & $1.9 \pm 0.14^{\mathrm{a}}$ & $1.71 \pm 0.15^{b}$ & $1.7 \pm 0.16^{b}$ \\
\hline & Tanks $^{2}$ & - & $2.38 \pm 0.2^{\mathrm{a}}$ & $2.2 \pm 0.15^{b}$ \\
\hline C22:4 n-6 $7,10,13,16$-docosatetraenoic & Tubs $^{1}$ & $3.26 \pm 0.16^{\mathrm{a}}$ & $3.37 \pm 0.25^{\mathrm{a}}$ & $3.35 \pm 0.24^{\mathrm{a}}$ \\
\hline & Tanks $^{2}$ & - & $2.93 \pm 0.22^{a}$ & $2.64 \pm 0.15^{a}$ \\
\hline C22:5 $n-3^{7,10,13,16,19-d o c o s a p e n t a e n o i c ~}$ & Tubs $^{1}$ & $0.9 \pm 0.08^{\mathrm{a}}$ & $1.01 \pm 0.12^{\mathrm{a}}$ & $0.95 \pm 0.15^{a}$ \\
\hline & Tanks $^{2}$ & - & $1.08 \pm 0.12^{\mathrm{a}}$ & $1.04 \pm 0.06^{\mathrm{a}}$ \\
\hline C22:5 n-6 $6^{4,7,10,13,16-\text { docosapentaenoic }}$ & Tubs $^{1}$ & $0.26 \pm 0.01^{\mathrm{a}}$ & $0.3 \pm 0.02^{\mathrm{a}}$ & $0.27 \pm 0.04^{\mathrm{a}}$ \\
\hline & Tanks $^{2}$ & - & $0.14 \pm 0.01^{a}$ & $0.13 \pm 0.01^{\mathrm{a}}$ \\
\hline $\mathrm{C} 22: 6 n-3^{\mathrm{DHA}}$ & Tubs $^{1}$ & $0.29 \pm 0.02^{a}$ & $0.26 \pm 0.03^{a}$ & $0.24 \pm 0.03^{a}$ \\
\hline & Tanks $^{2}$ & - & $0.52 \pm 0.06^{a}$ & $0.42 \pm 0.03^{b}$ \\
\hline
\end{tabular}


Table 5 (Continued)

\begin{tabular}{clccc}
\hline Fatty acid & Culture environment & Low density & Medium density & High density \\
\hline C24:1 $n-9$ & Tubs $^{1}$ & $0.07 \pm 0.0^{* *}$ & $0.03 \pm 0.02^{* *}$ & $0.03 \pm 0.02^{* *}$ \\
ARA : EPA : DHA ratio & Tanks $^{2}$ & - & $0.03 \pm 0.01^{\mathrm{a}}$ & $0.03 \pm 0.01^{\mathrm{a}}$ \\
& Tubs $^{1}$ & $1: 2.1: 0.2$ & $1: 2.3: 0.2$ & $1: 2.3: 0.2$ \\
& Tanks $^{2}$ & - & $1: 2.9: 0.3$ & $1: 3: 0.3$ \\
\hline
\end{tabular}

* Different superscripts within rows indicate significant $(P<0.05)$ differences.

** Significant $(P<0.05)$ interaction of density and size; see Table 6.

${ }^{1} n=4$ at low $\left(1000 \mathrm{~m}^{-2}\right)$, medium $\left(2000 \mathrm{~m}^{-2}\right)$ and high $\left(4000 \mathrm{~m}^{-2}\right)$ densities.

${ }^{2} n=6$ at medium $\left(2000 \mathrm{~m}^{-2}\right)$ and high $\left(6000 \mathrm{~m}^{-2}\right)$ densities.

Table 6 The interaction between culture density and graded size: mean $\left( \pm\right.$ SE) fatty acid contents* $\left(\mathrm{mg} \mathrm{g}^{-1}\right)$ of small $(\leq 0.6 \mathrm{~g})$ and large $(>0.6 \mathrm{~g})$ Perinereis helleri grown in polychaete-assisted sand filters in 2007. Data presented on a dry matter basis

\begin{tabular}{|c|c|c|c|c|c|c|c|}
\hline \multirow[b]{2}{*}{ Fatty acid } & \multirow{2}{*}{$\begin{array}{l}\text { Culture } \\
\text { environment }\end{array}$} & \multicolumn{2}{|l|}{ Low density } & \multicolumn{2}{|c|}{ Medium density } & \multicolumn{2}{|l|}{ High density } \\
\hline & & Small & Large & Small & Large & Small & Large \\
\hline \multicolumn{8}{|l|}{ Saturates } \\
\hline $\mathrm{C} 16: 0^{\text {palmitic }}$ & Tanks $^{2}$ & - & - & $6.07 \pm 0.3^{c}$ & $14.23 \pm 0.49^{a}$ & $4.64 \pm 0.36^{d}$ & $10.53 \pm 0.34^{b}$ \\
\hline C20:0 $0^{\text {arachidic }}$ & Tubs $^{1}$ & $0.08 \pm 0^{a}$ & $0.07 \pm 0.01^{\mathrm{ab}}$ & $0.06 \pm 0^{c}$ & $0.06 \pm 0^{\mathrm{bc}}$ & $0 \pm 0^{d}$ & $0.06 \pm 0^{c}$ \\
\hline \multicolumn{8}{|l|}{ Mono- and polyunsaturates } \\
\hline C18:1 $n-13^{5 \text {-octadecenoic }}$ & Tanks $^{2}$ & - & - & $1.4 \pm 0.07^{c}$ & $2.88 \pm 0.1^{\mathrm{a}}$ & $1.11 \pm 0.03^{d}$ & $2.15 \pm 0.05^{\mathrm{b}}$ \\
\hline C20:3 $n-3^{11,14,17-e i c o s a t r i e n o i c ~}$ & Tubs $^{1}$ & $0 \pm 0^{c}$ & $0.13 \pm 0.01^{\mathrm{a}}$ & $0 \pm 0^{c}$ & $0.06 \pm 0^{\mathrm{b}}$ & $0 \pm 0^{c}$ & $0 \pm 0^{c}$ \\
\hline$C 20: 5 n-3^{\mathrm{EPA}}$ & Tubs $^{1}$ & $3.41 \pm 0.02^{b c}$ & $3.8 \pm 0.03^{\mathrm{ab}}$ & $3.15 \pm 0.01^{c}$ & $3.92 \pm 0.13^{a}$ & $2.69 \pm 0.06^{d}$ & $3.85 \pm 0.23^{\mathrm{a}}$ \\
\hline $\mathrm{C} 24: 1 n-9^{\text {nervonic }}$ & Tubs $^{1}$ & $0.06 \pm 0.01^{\mathrm{b}}$ & $0.07 \pm 0^{\mathrm{a}}$ & $0 \pm 0^{c}$ & $0.06 \pm 0^{\mathrm{b}}$ & $0 \pm 0^{c}$ & $0.06 \pm 0^{\mathrm{b}}$ \\
\hline
\end{tabular}

* Different superscripts within rows indicate significant $(P<0.05)$ differences.

${ }^{1} n=2$ at low $\left(1000 \mathrm{~m}^{-2}\right)$, medium $\left(2000 \mathrm{~m}^{-2}\right)$ and high $\left(4000 \mathrm{~m}^{-2}\right)$ densities.

$2 n=3$ at medium $\left(2000 \mathrm{~m}^{-2}\right)$ and high $\left(6000 \mathrm{~m}^{-2}\right)$ densities.

Table 7 Mean $( \pm$ SE) proximate composition* of Perinereis helleri grown in polychaete-assisted sand filters in 2010 with (Farm A) and without (Farms A, B and C) fish meal supplemental feeding. Data presented as $\mathrm{g} \mathrm{kg}^{-1}$ dry matter

\begin{tabular}{lccrrr}
\hline & \multicolumn{3}{l}{ Farm A $^{1}$} & & \\
\cline { 2 - 3 } Component & Fed beds & Unfed beds & Farm B & Farm C \\
\cline { 2 - 4 } & & & & \\
\hline Moisture $^{3}$ & $779 \pm 2^{\mathrm{a}}$ & $791.5 \pm 7.5^{\mathrm{a}}$ & 817 & 795 \\
Ash & $104.5 \pm 2.5^{\mathrm{a}}$ & $118 \pm 4^{\mathrm{a}}$ & 140 & 123 \\
Energy $^{4}$ & $21.39 \pm 0.06^{\mathrm{a}}$ & $20.96 \pm 0.09^{\mathrm{a}}$ & 20.23 & 20.3 \\
Fat $^{5}$ & $73 \pm 3^{\mathrm{a}}$ & $86.5 \pm 5.5^{\mathrm{a}}$ & 61 & 50 \\
Total lipid $^{6}$ & $129.6 \pm 4^{\mathrm{a}}$ & $127.3 \pm 1.9^{\mathrm{a}}$ & 102.2 & 90.5 \\
Cholesterol & $8.69 \pm 0.11^{\mathrm{b}}$ & $9.68 \pm 0.05^{\mathrm{a}}$ & 10.32 & 8.58 \\
Nitrogen & $103.6 \pm 2.3^{\mathrm{a}}$ & $103.6 \pm 3.8^{\mathrm{a}}$ & 111.7 & 115.5 \\
Phosphorus & $7.7 \pm 0.2^{\mathrm{a}}$ & $7.8 \pm 0.2^{\mathrm{a}}$ & 8.2 & 7.8 \\
\hline
\end{tabular}

* Different superscripts within rows indicate significant $(P<0.05)$ differences.

$1 n=2$.

$2 n=1$.

${ }^{3} \mathrm{~g} \mathrm{~kg}^{-1}$ wet matter.

${ }^{4} \mathrm{MJ} \mathrm{kg}^{-1}$.

${ }^{5}$ Includes triglycerides, cholesterol and cholesterol esters.

${ }^{6}$ Includes free fatty acids, triacylglycerols and membrane-bound phospholipids. meal. Glutamic acid, glycine and serine occurred at higher $(P<0.05)$ levels in $P$. helleri from unfed beds, whilst tryptophan occurred at higher $(P<0.05)$ levels in those from fed beds. Glutamic acid had the highest representation of all amino acids across all farms ( $\left.85 \pm 2 \mathrm{~g} \mathrm{~kg}^{-1} \mathrm{DM}\right)$, followed by aspartic acid $\left(55 \pm 1 \mathrm{~g} \mathrm{~kg}^{-1}\right)$, glycine $\left(49 \pm 5 \mathrm{~g} \mathrm{~kg}^{-1}\right)$, arginine $\left(45 \pm 1 \mathrm{~g} \mathrm{~kg}^{-1}\right)$ and then alanine $\left(43 \pm 1 \mathrm{~g} \mathrm{~kg}^{-1}\right)$. In most cases, $P$. helleri from Farm A had lower levels of specific amino acids than those from Farms B and C.

\section{The prevalence of endemic shrimp viruses}

The pool of $P$. helleri sampled from the raceway-based PASF at BIRC in 2008 tested PCR-negative for GAV and IHHNV, but tested nested PCR-positive for MoV. A 301-nucleotide sequence obtained for the MoV-nested PCR amplicon possessed $100 \%$ identity to the sequence of MoV found in eastern Australian Penaeus monodon. P. helleri sampled from a similar raceway culture at BIRC in 2010 tested PCR-negative for all three shrimp viruses. 
Table 8 Mean $\left( \pm\right.$ SE) fatty acid contents* $\left(\mathrm{mg} \mathrm{g}^{-1}\right)$ of Perinereis helleri grown in polychaete-assisted sand filters in 2010 with (Farm A) and without (Farms A, B and C) fish meal supplemental feeding. Data presented on a dry matter basis

\begin{tabular}{|c|c|c|c|c|}
\hline \multirow[b]{2}{*}{ Fatty acid } & \multicolumn{2}{|l|}{ Farm $A^{1}$} & \multirow[b]{2}{*}{ Farm B ${ }^{2}$} & \multirow[b]{2}{*}{ Farm $C^{2}$} \\
\hline & Fed beds & Unfed beds & & \\
\hline \multicolumn{5}{|l|}{ Saturates } \\
\hline C14:0 & $0.67 \pm 0.06^{a}$ & $0.8 \pm 0.01^{a}$ & 0.21 & 0.13 \\
\hline C15:0 & $0.56 \pm 0.02^{a}$ & $0.61 \pm 0.01^{a}$ & 0.28 & 0.19 \\
\hline C16:0 palmitic & $16.94 \pm 0.3^{a}$ & $12.35 \pm 0.4^{\mathrm{b}}$ & 8.02 & 5.98 \\
\hline C17:0 & $1.74 \pm 0.07^{\mathrm{a}}$ & $1.97 \pm 0.03^{\mathrm{a}}$ & 1.17 & 0.81 \\
\hline C18:0 & $5.48 \pm 0.16^{a}$ & $4.63 \pm 0.04^{a}$ & 3.82 & 3.27 \\
\hline C19:0 & $0.2 \pm 0.01^{\mathrm{a}}$ & $0.18 \pm 0.02^{\mathrm{a}}$ & 0 & 0 \\
\hline C20: $0^{\text {arachidic }}$ & $0.4 \pm 0.02^{a}$ & $0.41 \pm 0.03^{a}$ & 0.43 & 0.9 \\
\hline C22:0 $0^{\text {behenic }}$ & $0.17 \pm 0.01^{a}$ & $0 \pm 0^{\mathrm{b}}$ & 0 & 0 \\
\hline \multicolumn{5}{|l|}{ Mono- and polyunsaturates } \\
\hline C16:1 n-7 & $2.76 \pm 0.19^{a}$ & $2.47 \pm 0.04^{a}$ & 1.0 & 0.68 \\
\hline C18:1 n-7 & $4.10 \pm 0.15^{\mathrm{a}}$ & $5.04 \pm 0.07^{\mathrm{a}}$ & 2.42 & 2.24 \\
\hline C18:1 n-9 & $3.19 \pm 0.14^{a}$ & $1.3 \pm 0.04^{b}$ & 0.85 & 0.64 \\
\hline C18:1 $n-13$ & $1.98 \pm 0.03^{a}$ & $1.97 \pm 0.06^{\mathrm{a}}$ & 1.68 & 1.38 \\
\hline C18:2 $n-6^{\text {linoleic }}$ & $1.84 \pm 0.11^{\mathrm{a}}$ & $0.92 \pm 0.02^{b}$ & 1.0 & 0.78 \\
\hline C18:3 n-3 & $1.37 \pm 0.11^{\mathrm{a}}$ & $1.39 \pm 0.21^{a}$ & 0.44 & 0.78 \\
\hline C18:4 n-3 & $0.49 \pm 0.02^{\mathrm{a}}$ & $0.17 \pm 0.01^{b}$ & 0 & 0 \\
\hline $\mathrm{C} 20: 1 n-7$ & $0.16 \pm 0.01^{a}$ & $0.34 \pm 0.04^{a}$ & 0 & 0.1 \\
\hline $\mathrm{C} 20: 1 n-9$ & $1.76 \pm 0.05^{a}$ & $1.16 \pm 0.04^{b}$ & 0.66 & 0.63 \\
\hline $\mathrm{C} 20: 1 \mathrm{n}-11^{9-\text { eicosenoic }}$ & $0.81 \pm 0.01^{b}$ & $1.12 \pm 0.02^{\mathrm{a}}$ & 0.94 & 0.5 \\
\hline $5,11-C 20: 2$ & $1.53 \pm 0^{\mathrm{a}}$ & $0.88 \pm 0.03^{b}$ & 0.41 & 0.32 \\
\hline$C 20: 2 n-6$ & $1.48 \pm 0.06^{a}$ & $1.14 \pm 0.06^{a}$ & 0.77 & 0.7 \\
\hline$C 20: 3 n-3$ & $0.14 \pm 0.01^{a}$ & $0.25 \pm 0.05^{a}$ & 0 & 0.1 \\
\hline C20:4 $n-6^{\text {ARA }}$ & $2.35 \pm 0.08^{\mathrm{a}}$ & $2.05 \pm 0.03^{a}$ & 2.31 & 1.97 \\
\hline $\mathrm{C} 20: 5 n-3^{\mathrm{EPA}}$ & $4.68 \pm 0.04^{a}$ & $5.61 \pm 0.16^{a}$ & 4.31 & 3.81 \\
\hline$C 22: 1 n-9$ & $0.15 \pm 0^{a}$ & $0.1 \pm 0^{\mathrm{b}}$ & 0 & 0 \\
\hline $7,13-C 22: 2^{\text {docosadienoic }}$ & $2.91 \pm 0.05^{\mathrm{a}}$ & $2.91 \pm 0.02^{a}$ & 3.01 & 1.93 \\
\hline C22:4 n-6 $7,10,13,16$-docosatetraenoic & $2.99 \pm 0.06^{b}$ & $3.89 \pm 0.1^{a}$ & 4.11 & 2.96 \\
\hline C22:5 $n-3^{7,10,13,16,19-d o c o s a p e n t a e n o i c}$ & $1.17 \pm 0.01^{\mathrm{b}}$ & $1.46 \pm 0.03^{a}$ & 1.0 & 0.89 \\
\hline$C 22: 5 n-6$ & $0.43 \pm 0^{\mathrm{a}}$ & $0.14 \pm 0^{\mathrm{b}}$ & 0 & 0.11 \\
\hline $\mathrm{C} 22: 6 n-3^{\mathrm{DHA}}$ & $2.64 \pm 0.02^{\mathrm{a}}$ & $0.62 \pm 0.06^{\mathrm{b}}$ & 0.43 & 0.52 \\
\hline C24:1 $n-9^{\text {nervonic }}$ & $0.31 \pm 0.01^{a}$ & $0 \pm 0^{\mathrm{b}}$ & 0 & 0 \\
\hline ARA : EPA : DHA ratio & $1: 2: 1.1$ & $1: 2.7: 0.3$ & $1: 1.9: 0.2$ & $1: 1.9: 0.3$ \\
\hline
\end{tabular}

* Different superscripts within rows indicate significant $(P<0.05)$ differences.

${ }^{1} n=2$.

$2 n=1$.

The pool of worms from several PASF beds at the $P$. monodon farm collected in 2009 was PCR-negative for GAV, IHHNV and MoV, as were worms collected in 2010 from each of three shrimp and fish farms. The positive and negative controls used in each PCR performed as expected supporting the validity of the data.

\section{Discussion}

Marine polychaete worms have been used for many years throughout the world for fishing bait and to condition shrimp and fish broodstock during maturation and prior to spawning (Makinouchi \& Primavera 1987; Olive 1999; Hoa et al. 2009; Chung et al. 2011). Wild fisheries have traditionally serviced these needs, but increasingly, polychaetes are commercially cultured for leisure fishing and marine hatchery applications (Olive 1999; Poltana et al. 2005; Palmer 2010; Limsuwatthanathamrong et al. 2012). Polychaete inclusions in shrimp maturation diets tend to range from 5 to 33\% (Meunpol et al. 2005; Coman et al. 2007), where they are preferred offered live, but for convenience are also supplied after thawing or in compounded forms. Despite their relatively high cost, their demand for use as bait and their functional advantages and preferences for use over other broodstock feeds have contributed to overexploitation, environmental impacts and market undersupply in several countries (Scaps 2003). Aquaculture offers an attractive solution to this, particularly as demonstrated 
Table 9 Mean $( \pm \mathrm{SE})$ amino acid contents* $\left(\mathrm{mg} \mathrm{g}^{-1}\right)$ of Perinereis helleri grown in polychaete-assisted sand filters in 2010 with (Farm A) and without (Farms A, B and C) fish meal supplemental feeding. Data presented on a dry matter basis

\begin{tabular}{|c|c|c|c|c|}
\hline \multirow[b]{2}{*}{ Amino acid } & \multicolumn{2}{|l|}{ Farm $A^{1}$} & \multirow{2}{*}{$\begin{array}{l}\text { Farm } \\
\mathrm{B}^{2}\end{array}$} & \multirow[b]{2}{*}{ Farm $C^{2}$} \\
\hline & Fed beds & Unfed beds & & \\
\hline Alanine & $42.04 \pm 0.5^{a}$ & $43.25 \pm 0.5^{a}$ & 45.89 & 38.68 \\
\hline Arginine & $43.48 \pm 1.25^{a}$ & $43.85 \pm 0.47^{a}$ & 49.97 & 45.36 \\
\hline Aspartic acid & $52.76 \pm 1.14^{a}$ & $54.52 \pm 0.95^{a}$ & 57.99 & 59.23 \\
\hline Cysteine & $7.75 \pm 0.1^{\mathrm{a}}$ & $7.76 \pm 0.03^{a}$ & 7.85 & 8.72 \\
\hline Glutamic acid & $79.71 \pm 1.22^{b}$ & $85.7 \pm 0.53^{a}$ & 94.32 & 87.89 \\
\hline Glycine & $37.6 \pm 0.35^{b}$ & $47.21 \pm 0.11^{a}$ & 55.86 & 69.14 \\
\hline Histidine & $13.74 \pm 0.13^{a}$ & $13.3 \pm 0.2^{a}$ & 14.8 & 14.36 \\
\hline Isoleucine & $21.99 \pm 0.01^{a}$ & $21.81 \pm 0.2^{a}$ & 25 & 24.22 \\
\hline Leucine & $38.67 \pm 0.24^{\mathrm{a}}$ & $38.34 \pm 0.43^{a}$ & 44.35 & 43.57 \\
\hline Lysine & $37.02 \pm 0.0^{\mathrm{a}}$ & $36.16 \pm 1.03^{a}$ & 43.35 & 40.2 \\
\hline Methionine & $12.23 \pm 0.3^{\mathrm{a}}$ & $11.34 \pm 0.36^{\mathrm{a}}$ & 11.8 & 13.79 \\
\hline Phenylalanine & $21.1 \pm 0.06^{a}$ & $20.69 \pm 0.29^{a}$ & 23.76 & 22.97 \\
\hline Proline & $25.38 \pm 0.16^{a}$ & $26.23 \pm 0.29^{a}$ & 27.92 & 28.13 \\
\hline Serine & $22.67 \pm 0.12^{b}$ & $24.75 \pm 0.16^{a}$ & 28.26 & 25.81 \\
\hline Threonine & $25.82 \pm 0.27^{\mathrm{a}}$ & $25.96 \pm 0.11^{\mathrm{a}}$ & 28.04 & 27.95 \\
\hline Tryptophan & $6.95 \pm 0.1^{a}$ & $6.22 \pm 0.08^{b}$ & 6.79 & 6.97 \\
\hline Tyrosine & $19.21 \pm 0.32^{\mathrm{a}}$ & $18.54 \pm 0.49^{a}$ & 21.53 & 20.37 \\
\hline Valine & $24.53 \pm 0.43^{a}$ & $24.46 \pm 0.34^{a}$ & 27.59 & 25.81 \\
\hline
\end{tabular}

* Different superscripts within rows indicate significant $(P<0.05)$ differences.

${ }^{1} n=2$.

${ }^{2} n=1$.

in this study within an integrated culture system where particular nutritional and biosecurity needs can be met with simple adjustments to culture and postharvest management. Furthermore, this localized conversion of waste nutrients into valuable and functional products has potential to provide environmental and economic benefits for farms and, importantly, the option to increase polychaete inclusions in maturation diets for higher breeding successes.

Polychaetes are generally reported to contain a healthy balance of nutrients and several factors that beneficially affect reproductive performance and larval quality in crustaceans. As in other previous studies, the present work found high concentrations of polyunsaturated fatty acids (particularly omega 3 and omega 6), which have important roles in membrane structures and metabolic processes and are precursors to biosynthetic pathways involved in the in vivo supply of sex steroids (Croz et al. 1988; Lytle et al. 1990; Izquierdo et al. 2001; Nguyen et al. 2012). For example, ARA was found here in P. helleri at relatively high levels. It is involved in the synthesis of prostaglandins which have a broad set of physiological roles including the regulation of reproduction in crustaceans (Harrison 1990; Wouters et al. 2001; Meunpol et al. 2010). Several polychaetes including some from this genus (Perinereis) have been shown to contain these and other hormones that stimulate ovarian development in one of the more difficult penaeid species to breed, P. monodon (e.g. prostaglandin: Poltana et al. 2005; progesterone, 17a-hydroxyprogesterone: Meunpol et al. 2007, 2010; methyl farnesoate: Laufer et al. 1997).

Reasonable proportions of lcPUFA, and particularly ARA and EPA, are thought critical for crustacean maturation diets (see Glencross 2009). Meunpol et al. (2005) documented a higher ratio of ARA compared with EPA (and DHA) in both sand (Perinereis sp.) and mud (Marphysa sp.) polychaetes that are commonly used for shrimp maturation in Thailand. That work demonstrated improvements in the condition of pond-reared male P. monodon fed diets modelled on this balance. Limsuwatthanathamrong et al. (2012) also recently reported higher proportions of ARA than that of EPA and DHA in P. nuntia grown on shrimp feed in Thailand. Other research has shown markedly different ratios within and between species (see summary in Table 10), yet many of these are reportedly also routinely used for shrimp broodstock with favourable results. For example, in the blood (or mud) worm Marphysa sanguinea, which has been much favoured by penaeid hatcheries in Queensland over the last 20 years, Marsden et al. (1992) found that ARA levels were about one-third lower than those of EPA.

In the present study, feeding $P$. helleri in PASF beds with fish meal significantly increased their DHA content. This offers a convenient means to adjust their nutritional profile especially because it also increases their biomass production (DAFF, unpublished data). However, it is yet to be determined whether this can provide nutritional advantages over the common practice of cofeeding shrimp with other fresh/frozen diets such as squid or green mussels that are naturally high in DHA (Marsden et al. 1992). Particular polychaete species that are not generally promoted for feeds in penaeid hatcheries, such as beachworms (Australonuphis sp.) and lugworms (Arenicola cristata), are reported to naturally have higher DHA than EPA (Lytle et al. 1990; Marsden et al. 1992), whereas polychaetes that are avidly promoted for this use tend to have much higher EPA ratios and sometimes to extreme (e.g. Nereis diversicolor: Bischoff et al. 2009; Glycera dibranchiata and N. viridens: Lytle et al. 1990) (see Table 10). Nereis diversicolor in particular has been studied in this regard by several researchers. It appears to have higher ratios of EPA under natural conditions, but reduced EPA relative to ARA and DHA when fed fish food or aquaculture sludge (GarciaAlonso et al. 2008; Bischoff et al. 2009). In the present 
Table 10 Ratios of ARA, EPA and DHA reported for various polychaete species under different environmental conditions

\begin{tabular}{|c|c|c|c|}
\hline Author & $\begin{array}{l}\text { Polychaete species common and } \\
\text { scientific names }\end{array}$ & Locality and environmental conditions & ARA/EPA/DHA ratio \\
\hline Farrington et al. (1973) & Catworm - Nephtys incisa & US, Rhode Island - natural & $1: 19.3: 11.8$ \\
\hline \multirow{4}{*}{ Lytle et al. (1990) } & Bloodworm - Glycera dibranchiata & US, Maine - natural & $1: 17.1: 3.5$ \\
\hline & Bloodworm - Americonuphis reesei & US, Panama - natural & $1: 3.8: 2.4$ \\
\hline & Lugworm - Arenicola cristata & US, Florida - natural & $1: 0.2: 0.3$ \\
\hline & Sandworm - Nereis viridens & US, Maine - natural & $1: 18.8: 2.1$ \\
\hline \multirow{2}{*}{ Marsden et al. (1992) } & Mudworm - Marphysa sanguinea & Queensland - natural* & $1: 1.5: 0.2$ \\
\hline & $\begin{array}{l}\text { Beachworm - Onuphidae } \\
\text { (Australonuphis sp.) }\end{array}$ & Queensland - natural* & $1: 2.1: 2.9$ \\
\hline \multirow[t]{4}{*}{ Luis \& Passos (1995) } & Ragworm - Nereis diversicolor & Portugal winter (Feb.) - natural & $1: 2.7: 0.2$ \\
\hline & & Portugal spring (May) - natural & $1: 2.6: 0.3$ \\
\hline & & Portugal summer (Aug.) - natural & $1: 2.5: 0.2$ \\
\hline & & Portugal autumn (Nov.) - natural & $1: 1.8: 0.1$ \\
\hline \multirow[t]{2}{*}{ Costa et al. (2000) } & Ragworm - Nereis diversicolor & Cultured - artificial fish feed & $?: 7.8: 4.9$ \\
\hline & & Cultured - soy feed & $?: 4.2: 0.7$ \\
\hline \multirow[t]{2}{*}{ Olive et al. (2002) } & King ragworm - Nereis virens & Cultured - terrestrial feed & $?: 7.7: 3.9$ \\
\hline & Lugworm - Arenicola marina & Cultured - terrestrial feed & $?: 1.5: 0.9$ \\
\hline \multirow[t]{2}{*}{ Meunpol et al. (2005) } & Sandworm - Perinereis sp. & Thailand - natural & $1: 1: 0.2$ \\
\hline & Mudworm - Marphysa sp. & Thailand - natural & $1: 0.6: 0.1$ \\
\hline \multirow[t]{6}{*}{ Garcia-Alonso et al. (2008) } & Ragworm - Nereis diversicolor & UK winter - natural & $1: 13.9: 1$ \\
\hline & & UK spring - natural & $1: 27.1: 2.7$ \\
\hline & & UK summer - natural & $1: 17.5: 1.3$ \\
\hline & & UK autumn - natural & $1: 13.3: 2.1$ \\
\hline & & Cultured - artificial fish feed & $1: 2.8: 0.8$ \\
\hline & & Cultured - eel sludge feed & $1: 5.2: 1.3$ \\
\hline \multirow[t]{2}{*}{ Bischoff et al. (2009) } & Ragworm - Nereis diversicolor & France - natural* & $0: 39: 0$ \\
\hline & & Cultured - integrated system* & $1: 4: 0.8$ \\
\hline Brown et al. (2011) & King ragworm - Nereis virens & Cultured - formulated worm feed* & $1: 6: 3$ \\
\hline \multirow{3}{*}{$\begin{array}{l}\text { Limsuwatthanathamrong } \\
\text { et al. (2012) }\end{array}$} & Sandworm - Perinereis nuntia & Thailand summer - natural & $1: 0.6: 0$ \\
\hline & & Thailand winter - natural & $1: 1.1: 0.4$ \\
\hline & & Cultured - shrimp feed & $1: 0.8: 0.6$ \\
\hline
\end{tabular}

Question marks indicate that this data was missing from the cited papers.

* Approximate ratios derived from graphical figures.

study, levels of EPA and ARA in fish-meal-fed P. helleri were not significantly reduced, but their DHA content was significantly increased. Those remarkably large ratio variations found in $N$. diversicolor between seasons and different environmental conditions suggest that their fatty acid contents may be more plastic and feed dependent than the nereidid species studied herein.

For most fatty acids in this study, general trends in results for different culture environments (i.e. tanks, tubs and farms) were similar. In this respect, size (as the result of physical sorting and mediated by density) had a more distinct influence on the nutritional contents of $P$. helleri. This is a different situation to that reported by Limsuwatthanathamrong et al. (2012) for cultured P. nuntia of different ages, because in the present study, different sized worms were the same age, but had grown at different rates due to competition and density effects within the same beds. Large P. helleri (and those grown at lower densities) tended to have higher fat and energy levels than small
P. helleri (and those grown at higher densities), and this was reflected in many specific fatty acid results. As with many other polychaete species, palmitic acid was the most prevalent saturate, followed by stearic acid. Palmitic acid is one of the most common fatty acids found in many animals, and it is the most prevalent fatty acid in the ovaries and nauplii of several penaeid species (Wouters et al. 2001), so can be considered an important component in a maturation diet for shrimp. Other than ARA, EPA and DHA, P. helleri also had reasonably high representations of many other mono- and polyunsaturated fatty acids with 16- to 24-carbon chain structures. These included some that are commonly found in other polychaete species (e.g. C18:1, C22:4, C22:5 classes) and some more specific ones that are not and are of general interest because they contained unusual configurations [e.g. six carbons between double bonds instead of the usual three in cis-5,cis-11-eicosadienoic acid (5,11-C20:2) and cis-7,cis-13-docosadienoic acid (7,13-C22:2)]. 
Regarding other classes of lipids, phospholipids (PLs) and sterols also have a wide range of important cellular functions in crustaceans including cytoplasm and membrane constituents and structures (Harrison 1990). Phospholipids and cholesterol have high predominance in mature shrimp ovaries and are therefore considered essential in maturation diets (Wouters et al. 2001). Both PLs that Wouters et al. (2001) identified as being predominant in shrimp ovaries (phosphatidylcholine and phosphatidylethanolamine) were well represented in approximately equal ratios in P. helleri, and the levels of total PLs (1.4$2 \%$ of DM) were in the order of the $2 \%$ of dietary intake recommended for shrimp broodstock by Cahu et al. (1994). This differed from P. nuntia in Thailand where only phosphatidylcholine was the main class identified (Limsuwatthanathamrong et al. 2012).

The dietary requirements for cholesterol by shrimp broodstock are less clear. Because it is a precursor to steroid hormones and comprises a large proportion of the lipid in crustacean eggs and because they cannot synthesize it or substantially replace it with other dietary sterols (Harrison 1990), it is likely that maturing broodstock would require greater levels than immature shrimp. Compared with levels of $0.3-0.4 \%$ recommended for diets of nonmaturing shrimp (Akiyama \& Dominy 1989) and inclusion levels ranging from 0.25 to $0.5 \%$ in maturation diets for P. vannamei (Cahu et al. 1994) and P. monodon (Meunpol et al. 2005) respectively, the levels found in $P$. helleri in the present study (5-10 $\mathrm{g} \mathrm{kg}^{-1}$ of DM) appear adequate. This was up to twice the cholesterol levels reported for cultured $P$. nuntia in Thailand (3-5 $\mathrm{g} \mathrm{kg}^{-1}$ of DM: Limsuwatthanathamrong et al. 2012).

When compared with other commercial polychaete species, P. helleri from PASF beds in this study had higher total lipid levels than others studied from Queensland, but lower levels than several from more temperate regions. $P$. helleri grown under commercially relevant conditions at three different farms returned dry matter fat and total lipid levels ranging from 50 to $90 \mathrm{~g} \mathrm{~kg}^{-1}$ and 90 to $130 \mathrm{~g} \mathrm{~kg}^{-1}$, respectively. This was similar to the lipid levels reported for Perinereis sp. by Meunpol et al. (2005) (140 $\left.\mathrm{g} \mathrm{kg}^{-1} \mathrm{DM}\right)$, higher than the lipid levels reported for wild Australonuphis sp. (30 $\left.\mathrm{g} \mathrm{kg}^{-1} \mathrm{DM}\right)$ and M. sanguinea (40 $\left.\mathrm{g} \mathrm{kg}^{-1} \mathrm{DM}\right)$ by Marsden et al. (1992) and for Marphysa sp. by Meunpol et al. (2005) (50 $\left.\mathrm{g} \mathrm{kg}^{-1} \mathrm{DM}\right)$, but lower than the respective fat and lipid levels reported for $N$. virens by Brown et al. (2011) (240 $\mathrm{g} \mathrm{kg}^{-1} \mathrm{DM}$ ) and Olive et al. (2002) (170 g kg $\mathrm{DM}$ ) and the maximum lipid levels reported for wild and cultured $N$. diversicolor by Luis \& Passos (1995)
(190 $\left.\mathrm{g} \mathrm{kg}^{-1} \mathrm{DM}\right)$, Costa et al. (2000) (200 $\left.\mathrm{g} \mathrm{kg}^{-1} \mathrm{DM}\right)$ and Garcia-Alonso et al. (2008) (320 $\left.\mathrm{g} \mathrm{kg}^{-1} \mathrm{DM}\right)$. As with $P$. helleri in this study, Pocock et al. (1971) also found that stage of maturity affected lipid accumulation and composition in $N$. virens, where ripening eggs accumulated triglycerides and glycerol ether esters. Levels of fat in maturing $P$. helleri females were more than twice that of small immature worms and three times the levels in maturing males, mainly due to an accumulation of palmitic and cis-vaccenic acids.

There is relatively less information for comparisons of other components. Carbohydrate levels $\left(\mathrm{g} \mathrm{kg}^{-1} \mathrm{DM}\right)$ in our P. helleri (60-80 $\mathrm{g} \mathrm{kg}^{-1}$ ) were lower than levels found by Brown et al. (2011) in N. virens (130-170 $\left.\mathrm{g} \mathrm{kg}^{-1}\right)$. All macrominerals identified by Akiyama \& Dominy (1989) for shrimp diets (calcium, phosphorus, potassium, magnesium sodium, sulphur) except chlorine and some microminerals (iron and zinc) were found at appreciable levels in $P$. helleri. Compared with $P$. helleri, levels they recommended for commercial shrimp feed were similar for zinc; higher for calcium and phosphorus; and lower for potassium, magnesium, sodium and iron.

For protein, P. helleri showed a tendency for higher protein levels in small compared with large worms (most noticeable in the 2007 data). On a dry matter basis, our small $P$. helleri had $670-690 \mathrm{~g} \mathrm{~kg}^{-1}$ protein (calculated from nitrogen contents), whilst large ones had 620$6460 \mathrm{~g} \mathrm{~kg}^{-1}$ protein. In comparison, Perinereis sp. in Thailand were reported by Meunpol et al. (2005) to have higher protein levels $(64 \%$ DM) than Marphysa sp. (510 $\left.\mathrm{g} \mathrm{kg}^{-1} \mathrm{DM}\right)$, whilst Marsden et al. (1992) reported $750 \mathrm{~g} \mathrm{~kg}^{-1}$ and $690 \mathrm{~g} \mathrm{~kg}^{-1}$ in Australonuphis sp. and M. sanguinea, respectively, and Brown et al. (2011) reported $570-620 \mathrm{~g} \mathrm{~kg}^{-1}$ in cultured $N$. virens. The protein/lipid ratio for our farm-raised P. helleri in 2010 (6:1) aligned well with that recommended for shrimp feed by Akiyama \& Dominy (1989), and the higher protein ratios from our earlier work in 2007 (up to $9: 1$ in small worms) suggest that $P$. helleri alone can provide adequate energy levels for shrimp. The amino acid contents of $P$. helleri in 2010 were similar to those reported by Marsden et al. (1992) for Australonuphis sp. and $M$. sanguinea, except that four of those five most heavily represented in $P$. helleri, namely glutamic acid, aspartic acid, glycine and alanine, were not recorded in that study. Arginine (45 $\mathrm{g} \mathrm{kg}^{-1} \mathrm{DM}$ ) was the fourth most prevalent amino acid in our $P$. helleri and the most prevalent amino acid found by Marsden et al. (1992) for both of those other species (59-60 $\mathrm{g} \mathrm{kg}^{-1} \mathrm{DM}$ ). 
Brominated phenols are other functional compounds that have been found in many polychaete species (Whitfield et al. 1999). They are thought to be used by lower marine organisms for deterrence and chemical defence and have also been found to have a range of other biological activities including antioxidant and antibacterial effects (Liu et al. 2011). For humans, low levels in food (1-10 $\mathrm{ng} \mathrm{g}^{-1}$ ) are considered desirable, but excessive levels are unpalatable, and their use in aquaculture feeds to enhance product flavour is a recent development (Fuller et al. 2008). Levels found in $P$. helleri in the present study were on the lower end of the very broad range of values found for many other species in eastern Australia (see Whitfield et al. 1999).

Because the endemic shrimp viruses GAV, MoV and IHHNV were not generally detected by PCR among batches of $P$. helleri collected from PASF beds at four different mariculture sites in Queensland, there appear good prospects for using worms reared in such systems as a dietary supplement for shrimp broodstock without them posing a virus transmission risk. Mourilyan virus, which is unlisted, but remains under review by the World Organisation for Animal Health (OIE Aquatic Animal Health Code, October 2011), was detected at low levels in P. helleri collected from BIRC in 2008. It can occur at high prevalence in P. monodon and P. japonicus in eastern Australia and is considered to be endemic throughout South-East Asia and the South Pacific (Cowley et al. 2005; Sellars et al. 2005). Although this is the first report of MoV occurring in a polychaete, interpretation of its significance requires caution as it was detected in only one sample of worms that had not been purged for long $(4 \mathrm{~h})$ and at low levels providing no confidence of infection. The surface-feeding nature of this worm species coupled with the surface concentration of pond detritus on PASF beds could be expected to present excellent opportunity for infection through ingestion of contaminants. Without productive viral infections establishing in the worms, purging them of their gut contents would be expected to remove such contaminants, and the long shelf-life of live P. helleri after harvest (several weeks) enhances this option. Extended purging durations $(>24 \mathrm{~h}$ ) thus appear a logical solution for reducing risks of viruses remaining in the guts of worms reared in PASF beds at shrimp farms where viral infections are commonplace. However, this detection of MoV in P. helleri suggests a need for future investigations into what purge durations may be useful for viral clearance and to confirm that they cannot support infections by problematic shrimp viruses.
This research documents the main nutritional contents of one intertidal polychaete species that can be successfully grown in PASF beds. Because P. helleri has performed better than $P$. nuntia in these developments in Queensland (Palmer 2010), it has been the focus of most research to date, but many other intertidal polychaete species may also be suitable for this application. In particular, P. helleri appears to have a well-balanced nutritional profile for shrimp and fish broodstock when grown in this way and offers a useful means to recover waste nutrients from landbased mariculture farms in the Indo-Pacific region. This work demonstrates new scope for the widespread production of polychaetes with specific nutritional contents and for various uses. It also demonstrates how PASF can provide controlled and sustainable supplies of this industrially important commodity and a viable alternative to diminishing wild polychaete resources.

\section{Acknowledgements}

This research was jointly supported by the Queensland Government's Department of Agriculture, Fisheries and Forestry through their Aquaculture Research Programme and by the Australian Government's Department of Agriculture, Fisheries Forestry through their National Landcare (Project No. 60945) and Caring for Our Country (Project No. SEQC1418) Programs. The authors wish to thank Richard Thaggard, Trevor Borchert, Catriona Morrison, Daniel Willett, Mick Markey, Kelli Anderson and Mahdi Green for technical support at BIRC and in the field. Also thanks to Michael Gravel, Adam Pytko, Peter Martin, Sharon Pun, Caterina Torrisi and Sue Poole for biomass analyses; David Mayer for biometry assistance; Ian Anderson for disease screening; and Jeff Cowley and Peter Lee for useful comments on the manuscript. We also gratefully acknowledge the on-farm support provided by the Bullock Creek Prawn Farm, Sunrise Seafoods and Creel Seafoods.

\section{References}

Akiyama, D.M. \& Dominy, W.G. (1989) Penaeid shrimp nutrition for the commercial feed industry. Texas Shrimp Farming Manual, Volume 1: Grow-out Technology, Technical Report of Texas Agricultural Extension Service and Texas A \& M University Sea Grant College Program. pp. 50.

AOAC (2000) Official Methods of Analysis of AOAC International, 17th edn. Association of Analytical Communities, Gaithersburg, MD, USA.

Avalli, A. \& Contarini, G. (2005) Determination of phospholipids in dairy products by SPE/HPLC/ELSD. J. Chromatogr. A, 1071, 185-190. 
Bischoff, A.A., Fink, P. \& Waller, U. (2009) The fatty acid composition of Nereis diversicolor cultured in an integrated recirculated system: possible implications for aquaculture. Aquaculture, 296, 271-276.

Brown, N., Eddy, S. \& Plaud, S. (2011) Utilization of waste from a marine recirculating fish culture system as a feed source for the polychaete worm, Nereis virens. Aquaculture, 322-323, 177-183.

Cahu, C., Guillaume, J.C., Stephan, G. \& Chim, L. (1994) Influence of phospholipid and highly unsaturated fatty acids on spawning rate and egg and tissue composition in Penaeus vannamei fed semi-purified diets. Aquaculture, 126, 159-170.

Christie, W.W. (2003) Lipid Analysis. Isolation, Separation, Identification and Structural Analysis of Lipids, 3rd edn. The Oily Press, Bridgwater.

Chung, M.-Y., Liu, C.-H., Chen, Y.-N. \& Cheng, W. (2011) Enhancing the reproductive performance of tiger shrimp, Penaeus monodon, by incorporating sodium alginate in the broodstock and larval diets. Aquaculture, 312, 180-184.

Coman, G.J., Arnold, S.J., Callaghan, T.R. \& Preston, N.P. (2007) Effect of two maturation diet combinations on reproductive performance of domesticated Penaeus monodon. Aquaculture, 263, 75-83.

Costa, P.F.E., Narciso, L. \& da Fonseca, L.C. (2000) Growth, survival and fatty acid profile of Nereis diversicolor (O.F. Muller, 1776) fed on six different diets. Bull. Mar. Sci., 67, 337-343.

Cowley, J.A., McCulloch, R.J., Rajendran, K.V., Cadogan, L.C., Spann, K.M. \& Walker, P.J. (2005) RT-nested PCR detection of Mourilyan virus in Australian Penaeus monodon and its tissue distribution in healthy and moribund prawns. Dis. Aquat. Org., 66, 91-104.

Croz, L., Wong, L., Justine, G. \& Gupta, M. (1988) Prostaglandins and related compounds from the polychaete worm Americonuphis reesei Fauchald (Onuphidae) as possible inducers of gonad maturation in Penaeid shrimps. Rev. Biol. Trop., 36, 331-332.

Farrington, J.W., Quinn, J.G. \& Davis, W.R. (1973) Fatty acid composition of Nephtys incisa and Yoldia limatula. J. Fish. Res. Board Canada, 30, 181-185.

Folch, J., Lees, M. \& Sloane Stanley, G.H. (1957) A simple method for the isolation and purification of total lipides from animal tissues. J. Biol. Chem., 226, 497-509.

Fuller, S.C., Frank, D.C., Fitzhenry, M.J., Smyth, H.E. \& Poole, S.E. (2008) Improved approach for analyzing bromophenols in seafood using stable isotope dilution analysis in combination with SPME. J. Agric. Food Chem., 56, 8248-8254.

Garcia-Alonso, J., Müller, C.T. \& Hardege, J.D. (2008) Influence of food regimes and seasonality on fatty acid composition in the ragworm. Aqua. Biol., 4, 7-13.

GenStat. (2011) GenStat for Windows, Release 14.1. VSN International Ltd., Oxford.

Glencross, B.D. (2009) Exploring the nutritional demand for essential fatty acids by aquaculture species. Rev. Aquacul., 1, 71-124.

Harrison, K.E. (1990) The role of nutrition in maturation, reproduction and embryonic development of decapod crustaceans: a review. J. Shellfish Res., 9, 1-28.

Hoa, N.D., Wouters, R., Wille, M., Thanh, V., Dong, T.K., Van Hao, N. \& Sorgeloos, P. (2009) A fresh-food maturation diet with an adequate HUFA composition for broodstock nutrition studies in black tiger shrimp Penaeus monodon (Fabricius, 1798). Aquaculture, 297, 116-121.

Izquierdo, M.S., Fernandez-Palacios, H. \& Tacon, A.G.J. (2001) Effect of broodstock nutrition on reproductive performance of fish. Aquaculture, 197, 25-42.
Kawahigashi, D.K. (1998) Overview of commercial maturation technology in the Western hemisphere. World Aquaculture Society Conference Proceedings, Brazil, 98, 381-392.

Kovacs, M.I.P., Anderson, W.E. \& Ackman, R.G. (1979) A simple method for the determination of cholesterol and some plant sterols in fishery-based food products. J. Food Sci., 44, 1299-1301, 1305.

Laufer, H., Paddon, J. \& Paddon, M. (1997)A hormone enhancing larva production in the pacific white shrimp, Penaeus vannamei. World Aquaculture Society Forth Symposium on Aquaculture in Central America: Focusing on Shrimp and Tilapia, Tegucigalpa, Honduras. pp. 161-162.

Limsuwatthanathamrong, M., Sooksai, S., Chunhabundit, S., Noitung, S., Ngamrojanavanich, N. \& Petsom, A. (2012) Fatty acid profile and lipid composition of farm-raised and wild-caught sandworms, Perinereis nuntia, the diet for marine shrimp broodstock. Asian J. Anim. Sci., 6, 65-75.

Liu, M., Hansen, P.E. \& Lin, X. (2011) Bromophenols in marine algae and their bioactivities. Mar. Drugs, 9, 1273-1292.

Luis, O.J. \& Passos, A.M. (1995) Seasonal changes in lipid content and composition of the polychaete Nereis (Hediste) diversicolor. Comp. Biochem. Physiol., 111B, 579-586.

Lytle, J.S., Lytle, T.F. \& Ogle, J.T. (1990) Polyunsaturated fatty acid profiles as a comparative tool in assessing maturation diets of Penaeus vannamei. Aquaculture, 89, 287-299.

Makinouchi, S. \& Primavera, J.H. (1987) Maturation and spawning of Penaeus indicus using different ablation methods. Aquaculture, 62, 73-81.

Marsden, G., McGuren, J.M., Sarac, H.Z., Neill, A.R., Brock, I.J. \& Palmer, C.L. (1992) Nutritional composition of some natural marine feeds used in prawn maturation. In: Proceedings of Aquaculture Nutrition Workshop, Salamander Bay, 15-17 April 1991 (Allan, G.L \& Dall, W. eds), pp. 82-86. NSW Fisheries, Brackish Water Fish Culture Research Station, Salamander Bay, Australia.

Marsden, G.E., McGuren, J.J., Hansford, S.W. \& Burke, M.J. (1997) A moist artificial diet for prawn broodstock: its effect on the variable reproductive performance of wild caught Penaeus monodon. Aquaculture, 149, 145-156.

Meunpol, O., Meejing, P. \& Piyatiratitivorakul, S. (2005) Maturation diet based on fatty acid content for male Penaeus monodon (Fabricius) broodstock. Aquacult. Res., 36, 1216-1225.

Meunpol, O., Iam-Pai, S., Suthikrai, W. \& Piyatiratitivorakul, S. (2007) Identification of progesterone and 17 $\alpha$-hydroxyprogesterone in polychaetes (Perinereis sp.) and the effects of hormone extracts on penaeid oocyte development in vitro. Aquaculture, 270, 485-492.

Meunpol, O., Duangjai, E., Yoonpun, R. \& Piyatiratitivorakul, S. (2010) Detection of prostaglandin $\mathrm{E}_{2}$ in polychaete Perinereis sp. and its effect on Penaeus monodon oocyte development in vitro. Fish. Sci., 76, 281-286.

Middleditch, B.S., Missler, S.R., Hines, H.B., McVey, J.B., Brown, A., Ward, D.G. \& Lawrence, A.L. (1980) Metabolic profiles of penaeid shrimp: dietary lipids and ovarian maturation. J. Chromatogr., 195, 359-368.

Nguyen, B.T., Koshio, S., Sakiyama, K., Ishikawa, M., Yokoyama, S. \& Kader, M.A. (2012) Effects of polychaete extracts on reproductive performance of kuruma shrimp, Marsupenaeus japonicus Bate. - Part II. Ovarian maturation and tissue lipid compositions. Aquaculture, 334-337, 65-72.

Okshima, T. (2001) Cholesterol. Current Protocols in Food Analytical Chemistry. John Wiley and Sons Inc., New York. 
Olive, P.J.W. (1999) Polychaete aquaculture and polychaete science: a mutual synergism. Hydrobiologia, 402, 175-183.

Olive, P.J.W., Craig, S., Cowin, P.B.D., Islam, M.D. \& Rutherford, G. (2002) The culture of Polychaeta as a contribution to sustainable production of aquafeeds. Aquaculture Europe 2001. Book of Abstracts European Aquaculture Society Special Publication, 32, 392-393.

Palmer, P.J. (2010) Polychaete-assisted sand filters. Aquaculture, 306, 369-377.

Palmer, P.J. \& Slattery, S.L. (2009) Using wheat flour in the production of organic banana prawns. In: Fisheries: Management, Economics and Perspectives (McManus, N.F. \& Bellinghouse, D.S. eds), pp. 313-334. Nova Science Publications, New York, USA.

Pocock, D.M.-E., Marsden, J.R. \& Hamilton, J.G. (1971) Lipids in an intertidal polychaete and their relation to maturation of the worm. Comp. Biochem. Physiol., 39A, 683-697.

Poltana, P., Lerkitkul, T., Anantasomboon, G., Wannapapho, W., Wongprasert, K., Olive, P.J.W. \& Withyachumnarnkul, B. (2005) Prostaglandins in the polychaete Perinereis nuntia and their receptors in the ovary of the black tiger shrimp Penaeus monodon. World Aquaculture Society Conference Proceedings, Bali.

Poltana, P., Lerkitkul, T., Pongtippatee-Taweepreda, P., Asuvapongpattana, S., Wongprasert, K., Sriurairatana, S., Chavadej, J., Sobhon, P., Olive, P.J.W. \& Withyachumnarnkul, B. (2007) Culture and development of the polychaete Perinereis cf. nuntia. Invertebr. Reprod. Dev., 50, 13-20.
Scaps, P. (2003) The exploitation and aquaculture of marine polychaetes. B Soc Zool Fr, 128, 21-33.

Sellars, M.J., Keys, S.J., Cowley, J.A., McCulloch, R.J. \& Preston, N.P. (2005) Association of Mourilyan virus with mortalities in farm-reared Penaeus (Marsupenaeus) japonicus transferred to maturation tank systems. Aquaculture, 252, 242-247.

Tang, K.F.J., Navarro, S.A. \& Lightner, D.V. (2007) PCR assay for discriminating between infectious hypodermal and hematopoietic necrosis virus (IHHNV) and virus-related sequences in the genome of Penaeus monodon. Dis. Aquat. Org., 74, 165-170.

Turchini, G.M., Torstensen, B.E. \& Ng, W.-K. (2009) Fish oil replacement in finfish nutrition. Rev. Aquacul., 1, 10-57.

Van Wijngaarden, D. (1967) Modified rapid preparation of fatty acid esters from lipids for gas chromatographic analysis. Anal. Chem., 39, 848-849.

Vijayan, K.K., Raj, V.S., Balasubramanian, C., Alavandi, S.V., Sekhar, V.T. \& Santiago, T.C. (2005) Polychaete worms - a vector for white spot syndrome virus (WSSV). Dis. Aquat. Org., 63, 107-111.

Whitfield, F.B., Drew, M., Helidoniotis, F. \& Svoronos, D. (1999) Distribution of bromophenols in species of marine polychaetes and bryozoans from eastern Australia and the role of such animals in the flavour of edible ocean fish and prawns (shrimp). $J$. Agric. Food Chem., 47, 4756-4762.

Wouters, R., Lavens, P., Nieto, J. \& Sorgeloos, P. (2001) Penaeid shrimp broodstock nutrition: an updated review on research and development. Aquaculture, 202, 1-21. 